\title{
The World-Wide Variation of Cosmic Ray Intensity by the Electro-Magnetic Field*
}

\author{
By Kazuo NAGASHIMA \\ Earth Science, Kyoto University
}

\begin{abstract}
The variation of cosmic ray intensity by the static electric field surrounding the earth was calculated in a previous paper.(1) In it a greater advantage was indicated in explaining the cosmic ray intensity decrease at the time of the magnetic storm by the electric field hypothesis than by the magnetic field hypothesis suggested by $\mathrm{S}$. Chapman. ${ }^{(2)}$ In the present paper, the intensity variations of the ionizing and neutron components produced by the variation of the static electric field are calculated in detail extending over various altitudes and latitudes. By comparing these calculations with observations, it can be concluded that this electric field hypothesis is possible in explaining the phenomenon of the world-wide variations in intensity pointed out recently by various authors. ${ }^{(3),(4)}$

It may be possible that the diurnal variation of cosmic ray intensity can be produced by an electric field not derived from potential.
\end{abstract}

\section{Introduction}

Up to date, various types of cosmic ray intensity variation have been reported. Among these, the present paper is concerned with the following:

[A] The world-wide variation of cosmic ray intensity (not dependent on local time). This involves the following phenomena:

(a) The world-wide decrease of cosmic ray intensity at the time of the cosmic ray storm..$^{(5)}$

(b) The world-wide fluctuations of cosmic ray intensity.(3),(4)

[B] The diurnal variation of cosmic ray intensity.

In order to explain phenomenon [A], any theory or hypothesis must correspond the following conditions:

[I] The variation is world-wide.

[II] The latitude dependence of the variation.

Based on his experiments, ${ }^{(3)}$ H.V. Neher pointed out that:

(1) The changes in the ionizing component at sea level are nearly independent of latitude.

* Contributed to Geophysical Papers dedicated to Prof. M. Hasegawa on his sixtieth birthday. 
(2) The changes in the ionizing component increase with the increase of latitude.

[III] The altitude dependence of the variation.

(1) The fluctuations in the ionizing component at high altitudes are larger than at intermediate altitudes or at ground level.(3)

(2) The changes in the neutron component are several times those of the ionizing component. ${ }^{(3),(6),(23)}$

It was pointed out in a previous paper(1) that the variation of electric potential on the earth causes such world-wide variations in cosmic ray intensity, and suggested that the comparison between the variations at different stations would be a key towards determining the true cause of the production of such a world-wide variation. Recently, according to this electric field hypothesis, W.H. Fonger explained the phenomenon (2) in [III]. ${ }^{(6)}$ But, to explain all the above mentioned phenomena by this electric field hypothesis, it is necessary to calculate the cosmic ray intensity variations, produced by the electric field, over various altitudes and latitudes, more precisely.

In $\S 1$, the general formula of the cosmic ray intensity variation which is produced by the static electric and magnetic field is discussed. Generally, in such a static electric and magnetic field, the cosmic ray intensity variation is due not only to the variation of the primary differential intensity spectrum caused by the variation of the static electric field but also to the variation of the cut-off energy (see $\S 1$ ) caused by the variation of the static electric and magnetic field. In $\S 2$, neglecting the influence of the variation of cut-off energy, there is calculated the intensity variation which is produced by the variation of the static electric field. The variation of cut-off energy cannot be determined unless the state of the static electric and magnetic field surrounding the earth is known. In case of a simple model of the field, the variation can be obtained analytically and is discussed in App. I. But the altitude curve of the intensity variation caused by the variation of cut-off energy can be obtained without such a model of the field and is discussed in $\S 3$. In $\S 4$, comparisons of the intensity variations calculated theoretically in $\S 2$ and $\S 3$ are made with those obtained by observation.

The diurnal variation of cosmic ray intensity is a world-wide phenomenon which is dependent on local time. The solar magnetic field theory was advocated by M.S. Vallarta ${ }^{(7)}$ to explain this phenomenon, but at low latitudes this theory seems to be inadequate. In $\S 5$, the possibility of explaining this phenomenon by the electric field hypothesis is discussed, although the origin of the electric field cannot be pointed out.

\section{§ 1. The General Formula of Cosmic Ray Intensity Variation by the Static Electric and Magnetic Field}

Let $N(\lambda, x)$ denote the directional cosmic ray intensity, observed at geomagnetic latitude $\lambda^{\circ}$ and atmospheric depth $x \mathrm{~g} \mathrm{~cm}^{-2}$, and the following relation holds at the normal state; 


$$
N(\lambda, x)=\sum_{A} \int_{E_{\lambda, A}, Z}^{\infty} m_{A, Z}(E, x) \cdot i_{A, Z}(E) d E ; \cdots \cdots
$$

where $i_{A, Z}(E)$ is the primary differential intensity spectrum of the nucleus, its atomic number $A$ and its charge number $Z$, and $E$ is the kinetic energy per nucleon. $m_{A, Z}(E, x)$ is the number of secondary particles at $x \mathrm{~g} \mathrm{~cm}^{-2}$ produced by one of the primary cosmic rays whose kinetic energy is $E$ per nucleon and called the over-all multiplicity. ${ }^{(8)} \quad E_{\lambda, A, Z}$ denotes the minimum kinetic energy per nucleon of primary particle which can be injected upon the earth at $\lambda^{\circ}$ from a given direction and is called the cut-off energy.

Strictly speaking, the formula of Eq. (1) is not correct because it may happen that the primary spectrum might in some way be interrupted in some energy region, and consequently $N(\lambda, x)$ cannot be expressed by only the upper and lower limits of integral in Eq. (1). For example, such a situation occurs when we intend to explain the diurnal variation of cosmic ray intensity by the solar magnetic field. ${ }^{(7)}{ }^{(9)}$ In such a case, however, Eq. (1) can be easily expanded. The case chiefly discussed here is one where Eq. (1) holds.

If the state of the static electric and magnetic field surrounding the earth varies from the normal state, then, by the influence of this variation, the primary cosmic ray, reaching the earth, loses its energy to an amount of $\Delta E$ per nucleon, where

$$
\Delta E=\frac{Z}{A} e \Delta \phi
$$

$\Delta \phi$ is the variation of the earth's electric potential relative to the normal state. In this case, Eq. (1) changes as follows,

$$
N\left(\lambda, x, \Delta E, \delta E_{\lambda}\right)=\sum_{A} \int_{E_{\lambda, A, Z} Z^{+\delta E_{\lambda, A} Z}}^{\infty} m_{A, Z}(E, x)[1-L(E+\Delta E, \Delta E)] \cdot i_{A, Z}(E+\Delta E) d E, \cdots(
$$

where $L(E, \Delta E)$ is Liouville's effect(1),(10) and given by Eq. (4).

$$
L(E, \Delta E)=\frac{2\left(E+m_{0} c^{2}\right) \Delta E-(\Delta E)^{2}}{\left(E+m_{0} c^{2}\right)^{2}-\left(m_{0} c^{2}\right)^{2}}, \cdots \cdots \cdots \cdots
$$

where $m_{0} c^{2}$ is the rest energy per nucleon. In Eq. (3), $\delta E_{\lambda_{1}, A_{1} Z}$ is the variation of cut-off energy, which is caused not only by the variation of the magnetic field but also by the variation of the electric field and cannot be uniquely determined unless the state of the electro-magnetic field is determined. $\delta E_{\lambda}$ in the parenthesis on the left hand side of Eq. (3) denotes the occurrence of the variation of cut-off energy. On the contrary, the variation of the primary spectrum in Eq. (3) is determined if only the electric field is derived from potential ${ }^{(12)}$ and is independent of the form of the static electric and magnetic field. More generally, the static magnetic field which is not symmetrical to the earth's magnetic dipole axis could change the primary spectrum in the low energy region. For example, we see that the solar magnetic field produces such a situation if we consider that, as pointed out above, the solar magnetic field $^{(7),(9)}$ has an agency to interrupt the primary spectrum in some energy region, and 
moreover that the primary particles exist in some degree in such an interrupted energy region of the primary spectrum because, as pointed out by $\mathrm{H}$. Alfven ${ }^{(13)}$ and and J.A. Wheeler et al., (14) there exist the primary particles in the forbidden region of the solar magnetic field. The intensity variation produced by such a static magnetic field depends on local time, so we will discuss this in $\S 5$, and not consider it here.

The intensity variation relative to the normal state is defined by

$$
y(\lambda, x, \Delta E, \delta E)=\frac{N(\lambda, x)-N\left(\lambda, x, \Delta E, \delta E_{\lambda}\right)}{N(\lambda, x)} . \cdots \cdots \cdots
$$

As seen from Eqs. (5) and (3), $y$ is not proportional to $\Delta E$.(11) $^{(1)}$

As mentioned above, the causes which produce the variation $y$, are divided into two classes as follows: (1) the variation of primary spectrum which is produced by the variation of electric potential and (2) the variation of cut-off energy which is produced by the variation of static electric and magnetic field. Hereafter, an examination is to be made of the variation $y$ by these two causes individually.

\section{$\S 2$. The Cosmic Ray Intensity Variation by the Static Electric Field (not considering the Variation of Cut-Off Energy)}

Recently, the existence of heavy particles in the primary cosmic rays has been pointed out.(16) But, as their intensity spectrums in the high energy region are not yet well known, we assume that all the primary particles consist of protons only and adopt as its intensity spectrum that produced by H.V. Neher ${ }^{(3),(16)}$

$$
\left.\begin{array}{rlrl}
i(E) d E & =\frac{0.048}{E^{2 / 3}\left(1+0.09 E^{4 / 3}\right)^{3 / 2}} d E, & & \left(E \geq E_{g}=0.8 \text { Bev. }\right) \\
& =0, & \left(E<E_{g}\right)
\end{array}\right\} \ldots \ldots
$$

where $E_{g}$ is the energy, in the lower energy region than which the primary particles do not exist, and is called hereafter the general cut-off energy to avoid being confused with the cut-off energy $E_{\lambda}$. This spectrum leads to a smaller absolute value of intensity compared to that observed by others. ${ }^{(17)}$ But, it only is necessary that the distribution of the primary spectrum is correct and it is not necessary that the absolute value of intensity is correct, because the variation $y$ in Eq. (5) depends only upon the distribution, but not upon the absolute value. In this point, it is reasonable to adopt the above spectrum.

Neglecting the variation of cut-off energy, and considering only the vertical cosmic ray intensity, Eqs. (1) and (3) becomes as follows,

$$
\begin{aligned}
N(\lambda, x) & =\int_{E_{\lambda}}^{\infty} m(E, x) \cdot i(E) d E, \quad \cdots \cdots \cdots \\
N(\lambda, x, \Delta E, 0) & =\int_{E_{\lambda}}^{\infty} m(E, x)[1-L(E+\Delta E, \Delta E)] \cdot i(E+\Delta E) d E,
\end{aligned}
$$

where $E_{\lambda}$ denotes the cut-off energy of cosmic rays which are incident upon the earth at $\lambda^{\circ}$ from the vertical direction. To calculate $y$ in Eq. (5), it is necessary to know the over-all multiplicity $m(E, x)$. By making an assumption that $m(E, x)$ is proportional to $E^{a}$, where $a$ is constant, it is possible to obtain $m(E, x)$ of the ionizing and 
neutron components by using the observations made by H.V. Neher and J.A. Simpson et al., respectively (see App. II). The results obtained are shown in Figs. 1 and 2, and in Table I. Two different $m(E, 1030)$ of the ionizing component are tabulated in Table I. These over-all multiplicities are those of the two extreme cases estimated on the basis of assumption (see App. II). The full lines in Figs. 1 and 2 denote the over-all multiplicities obtained directly from the observations without any assumption and $E_{c}$ in Table $\mathrm{I}$ is the critical energy at which the distribution of $m(E, x)$ changes.
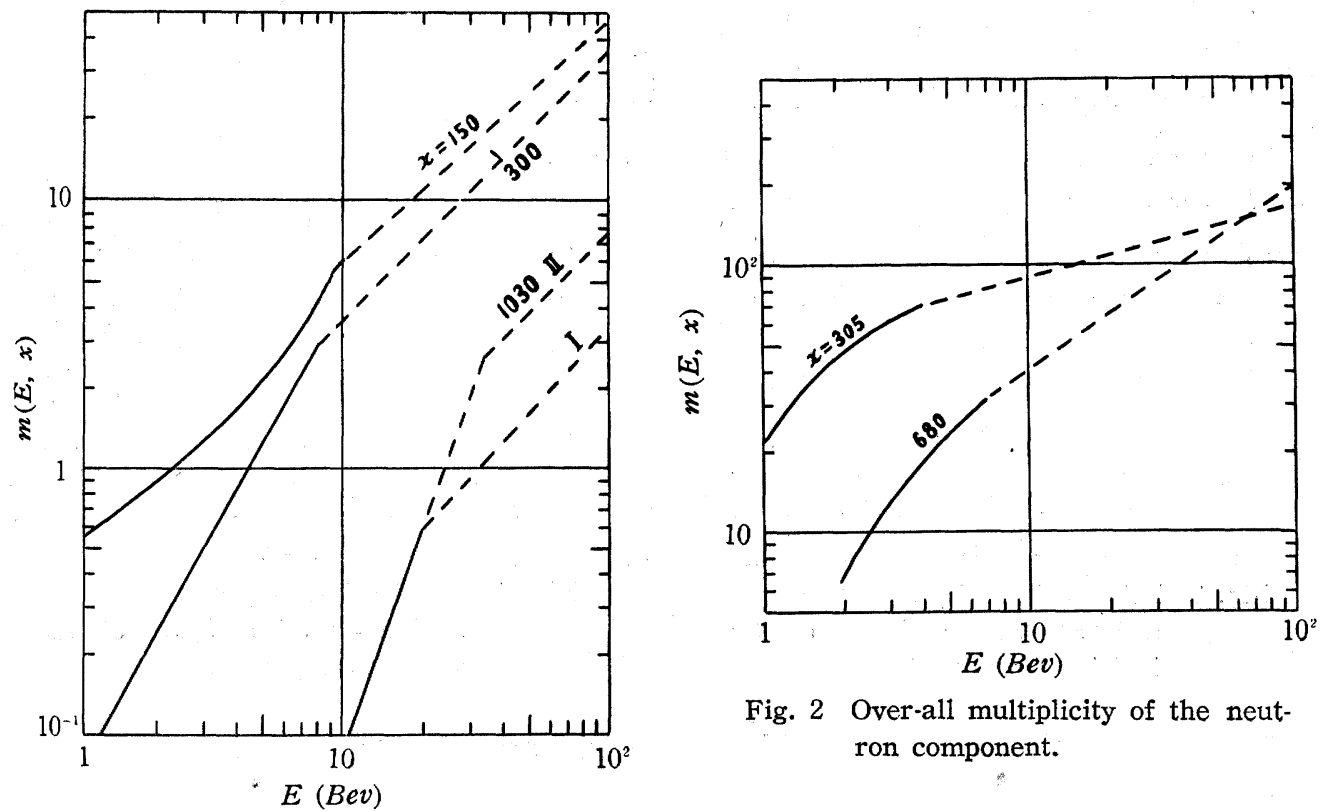

Fig. 2 Over-all multiplicity of the neutron component.

Fig. 1 Over-all multiplicity of the ionizing component.

Table I $m(E, x)$

\begin{tabular}{|c|c|c|c|c|c|}
\hline & \multicolumn{2}{|c|}{$x\left(\mathrm{~g} \mathrm{~cm}^{-2}\right)$} & $m(E, x)\left(E<E_{c}\right)$ & $E_{c}($ Bev.) & $m(E, x)\left(E \geqq E_{c}\right)$ \\
\hline \multirow{5}{*}{. } & \multicolumn{2}{|c|}{0} & 1 & & 1 \\
\hline & \multicolumn{2}{|c|}{150} & See Fig. 1. & 10 & $6.1 \cdot\left(\frac{E}{E}\right)^{0.9}$ \\
\hline & \multicolumn{2}{|c|}{300} & $7.4 \cdot 10^{-2} \cdot E^{1.8}$ & 8.2 & $3.0 \cdot\left(\frac{E}{E}\right)^{1.0}$ \\
\hline & \multirow{2}{*}{1030} & I & $1.3 \cdot 10^{-4} \cdot E^{2,8}$ & 15 & $2.8 \cdot 10^{-1} \cdot\left(\frac{E}{E_{c}}\right)^{1.3}$ \\
\hline & & II & $1.3 \cdot 10^{-4} \cdot E^{2.8}$ & 23.1 & $9.5 \cdot 10^{-1} \cdot\left(\frac{E}{E_{n}}\right)^{1.0}$ \\
\hline \multirow{2}{*}{ 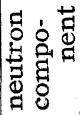 } & \multicolumn{2}{|c|}{305} & See Fig, 2. & 4 & $50 \cdot E^{0.26}$ \\
\hline & \multicolumn{2}{|c|}{680} & See Fig. 2. & 7 & $8.6 \cdot E^{0.68}$ \\
\hline
\end{tabular}

* (This over-all multiplicity was oftained from the latitude curve of intensity normalized to unity at the equator.)

In Figs. 3 and 4 , the functions of $n(E, x)=m(E, x) i(E)$ of the ionizing and neutron components are shown respectively and normalized to unity at $E=10 \mathrm{Bev}$. to facilitate their mutual comparișon, 


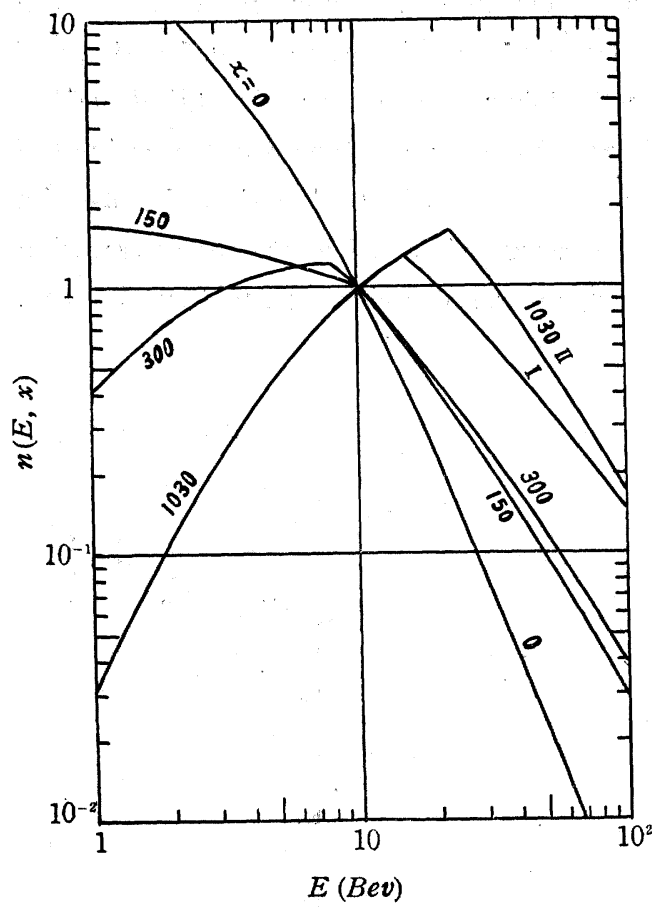

Fig. 3 Relative intensity spectrum, $n(E, x)=m(E, x) \cdot i(E)$, of the ionizing component.

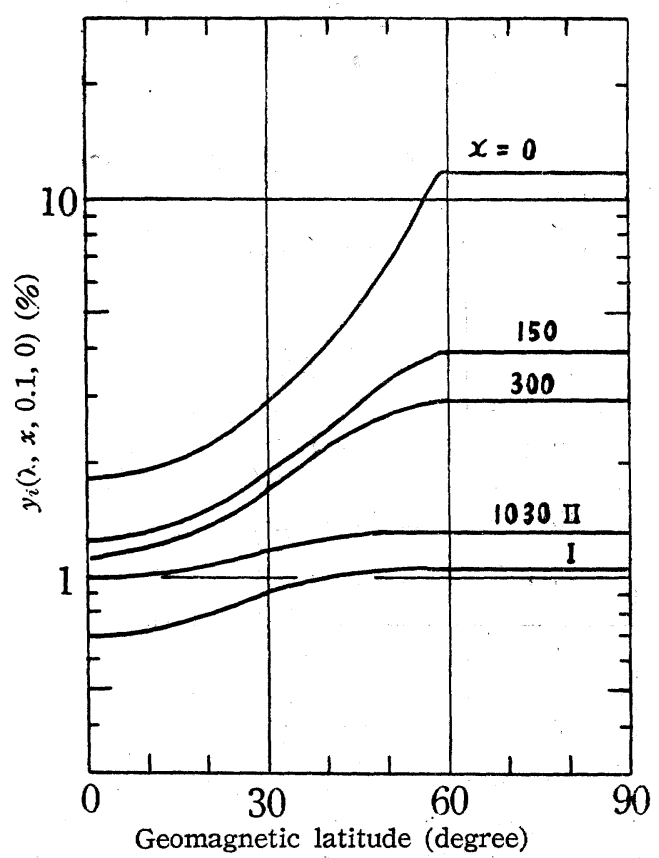

Fig. 5 Latitude dependence of $y$ of the ionizing component by the variation of the static electric field.

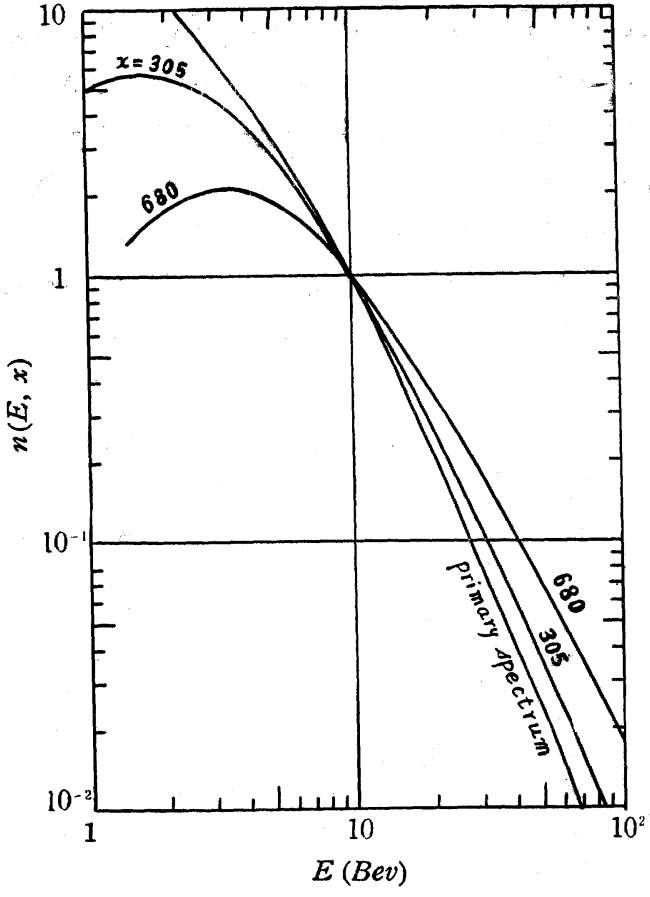

Fig. 4 Relative intensity spectrum, $n(E, x)=m(E, x) \cdot i(E)$, of the neutron component.

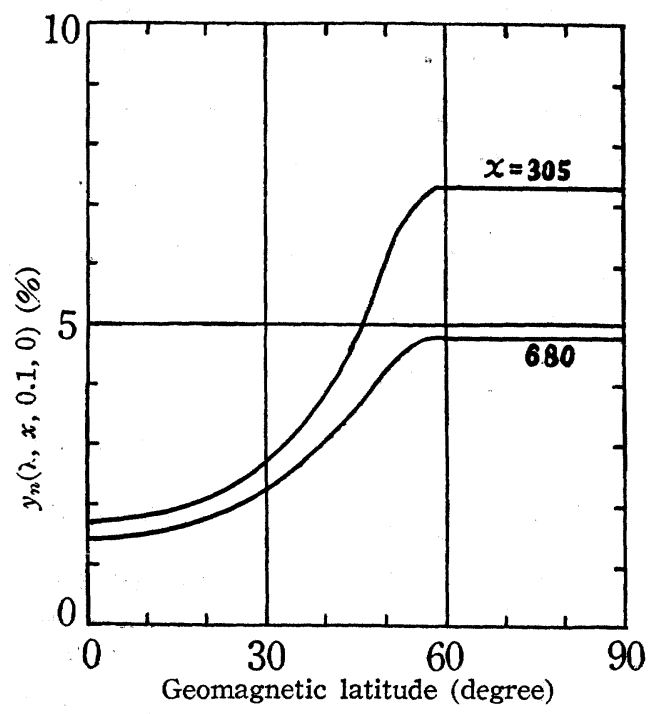

Fig. 6 Latitude depeddence of $y$ of the neutron component by the variation of static electric field. 
By using these over-all multiplicities and the primary spectrum given by Eq. (6), it is possible to calculate $y(\lambda, x, \Delta E, 0)$. Figs. 5 and 6 show the latitude curves of $y$ of the ionizing and neutron components at various altitudes in case $\Delta E=0.1 \mathrm{Bev}$. In these figures, the subscripts $i$ and $n$ of $y$ represent the ionizing and neutron components respectively.

The altitude curves of $y$ at various latitudes are obtained by drawing the smoothed curves through the calculated points and shown in Figs. 7 and 8 . In Fig. 7, the curves of the ionizing component are normalized to unity at $x=0$.

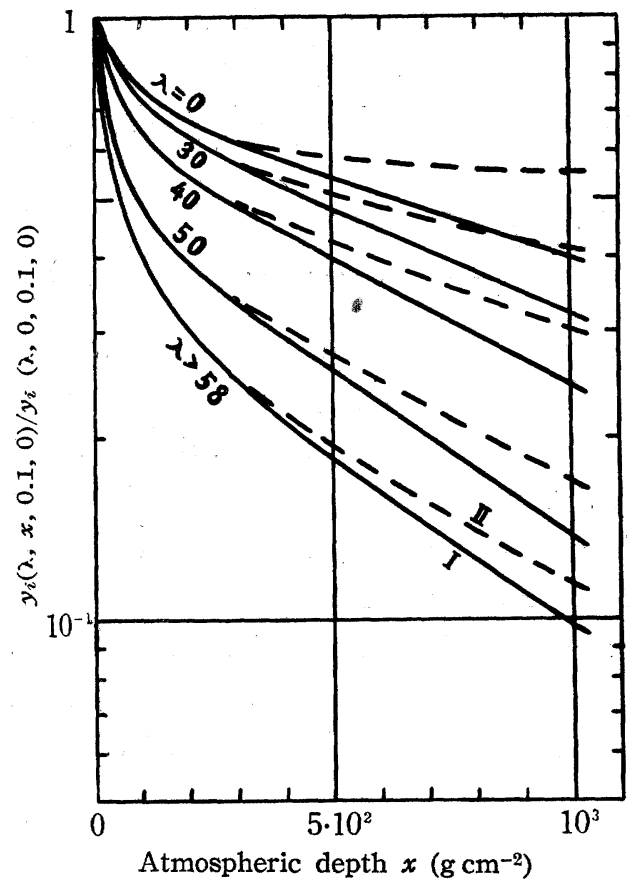

Fig. 7 Altitude dependence of $y$ of the ionizing component by the variation of the static electric field.

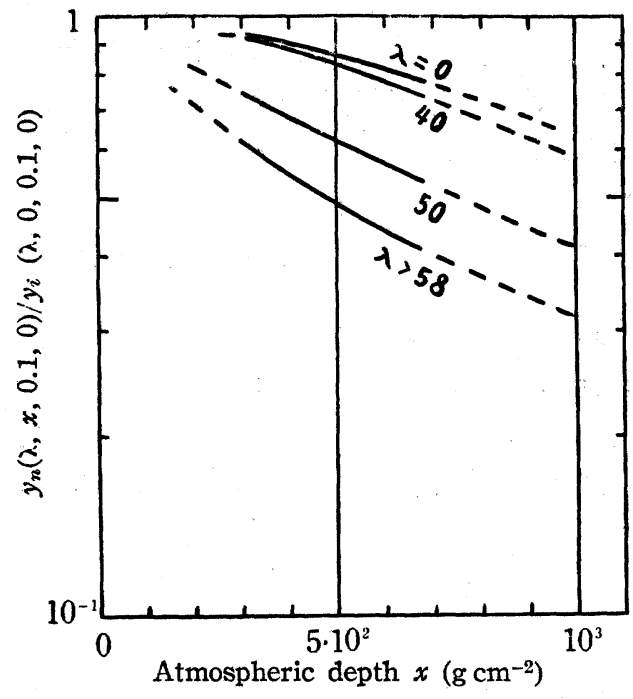

Fig. 8 Altitude dependence or ' $y$ of the neutron component by the variation of the static electric field.

The variation of the neutron component is produced by the variation of the primary ionizing particles which is caused by the variation of the electric field, so that the curves of the neutron component are normalized so as to satisfy the condition that the variation of the primary particles becomes just to unity. J.A. Simpson et al. (18). reported that, in the atmospheric depth greater than $600 \mathrm{~g} \mathrm{~cm}^{-2}$, there were no differences in the latitude dependence of the neutron intensity. If so, the variation $y$ of the neutron component must be independent on altitude in such a region. On the contrary, Fig. 8 obtained by drawing the smoothed curves through the calculated points shows that $y$ decreases slowly with the increase of $x$ in such a region. This discrepancy between the theoretical and experimentally expected curves might be due to a lack of the calculated points, 


\section{§ 3. The Cosmic Ray Intensity Variation by the Variation of Cut-Off Energy}

As discussed in $\S 1$, the variation of cut-off energy is another cause which produces the cosmic ray intensity variation, and cannot be uniquely determined unless the state of the static electric and magnetic field surrounding the earth is determined. Even if the state is determined, it is still difficult to obtain the variation of cut-off energy. In App. I, this problem is treated in a simple case when the static electric and magnetic field is symmetrical to the earth's magnetic dipole axis.

The latitude curve of $y$ cannot be obtained when the variation of cut-off energy is not known as a function of latitude, but it is possible to obtain the altitude curve of $y$ if only $N(\lambda, x)$ can be known from the observations. If the cut-off energy $E_{\lambda}$ changes to $E_{\lambda}+\delta E_{\lambda}$ by the influence of the static electric and magnetic field, $y$ in Eq.

(5) becomes as follows,

$$
y\left(\lambda, x, 0, \delta E_{\lambda}\right)=\frac{\int_{E_{\lambda}}^{E_{\lambda}+\delta E_{\lambda}} m(E, x) i(E) d E}{N(\lambda, x)} \ldots \ldots
$$

Figs. 9 and 10 represent the variation $y$ at various altitudes and latitudes in case $\delta E_{\lambda}=0.1 B e v$. Care must be taken that, as mentioned above, these curves do not represent those of the latitude effect of $y$, different from those in Figs. 5 and 6 . At high latitudes, $y=0$ as shown in Figs. 9 and 10 . This phenomenon is partly due to the fact that there are no primary cosmic rays in the lower energy region other than the general cut-off energy $E_{g}$ and partly to the fact that at the low atmosphere there is the knee of the latitude effect of cosmic ray intensity produced by atmospheric absorption. But if $\delta E_{\lambda}$ becomes very large under the influence of some mechanism even in such high latitudes, the variation $y$ occurs as well as at low latitudes.

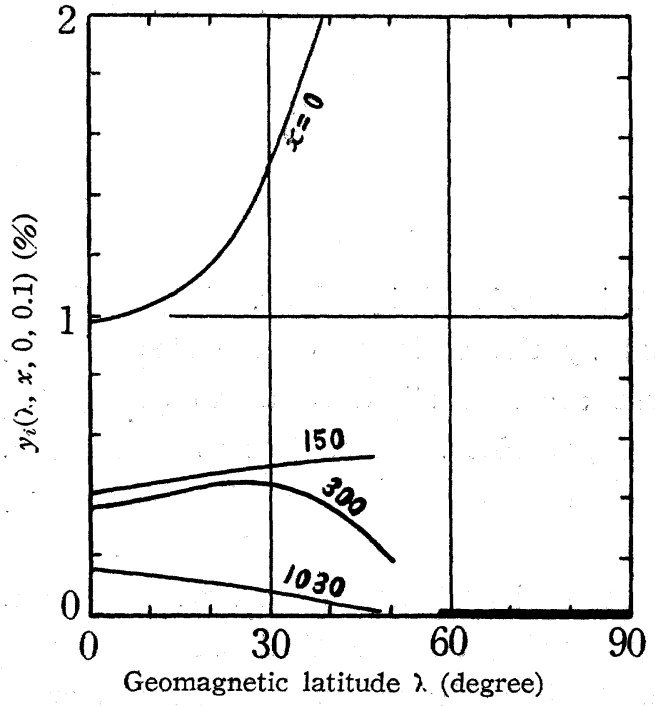

Fig. 9 Variation, $y$, of the ionizing component by the variation of the cut-off energy.

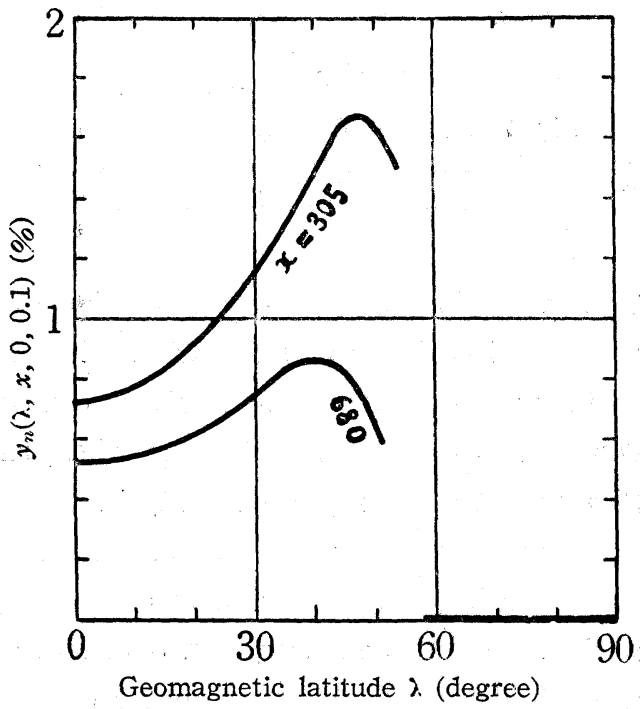

Fig. 10 Variation, $y$, of the neutron component by the variation of the cut-off energy. 
Figs. 11 and 12 show the altitude curves of $y$ produced by the variation of cutoff energy. The normalization is quite similar to that adopted in case of Figs. 7 and 8. As seen from Figs. 7, 8, 11 and 12, the altitude curves, in case of the variation of cut-off energy, decrease more rapidly with the increase of $x$, compared with those in case of the variation of electric field. Such a difference is caused by the fact that, in the former case, only the low energy particles are excluded; in the latter case, on the contrary, all the particles are influenced by the electric field.

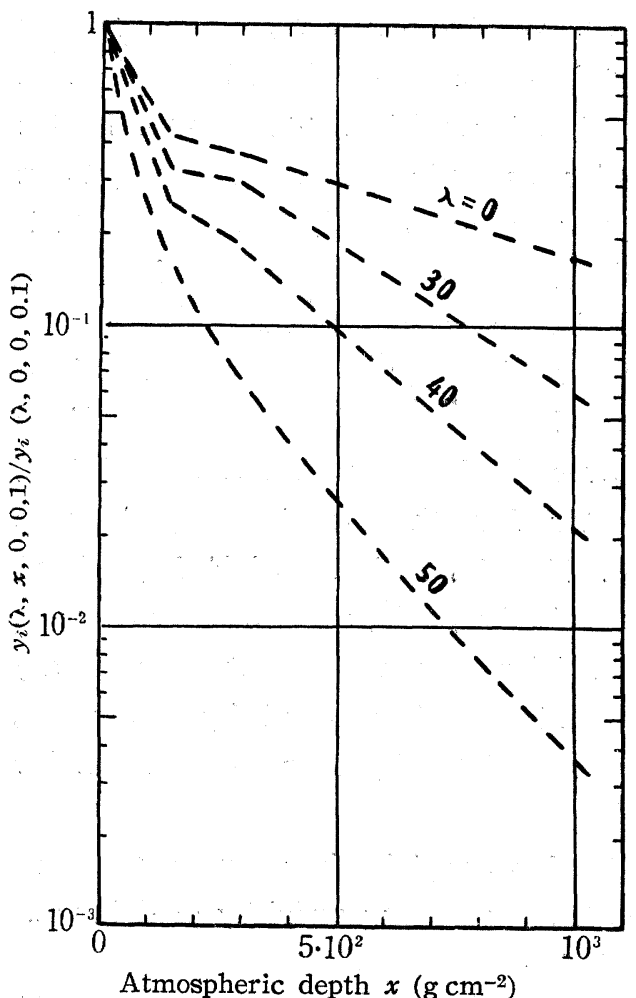

Fig. 11 Altitude dependence of $y$ of the ionizing component by the variation of the cut-off energy.

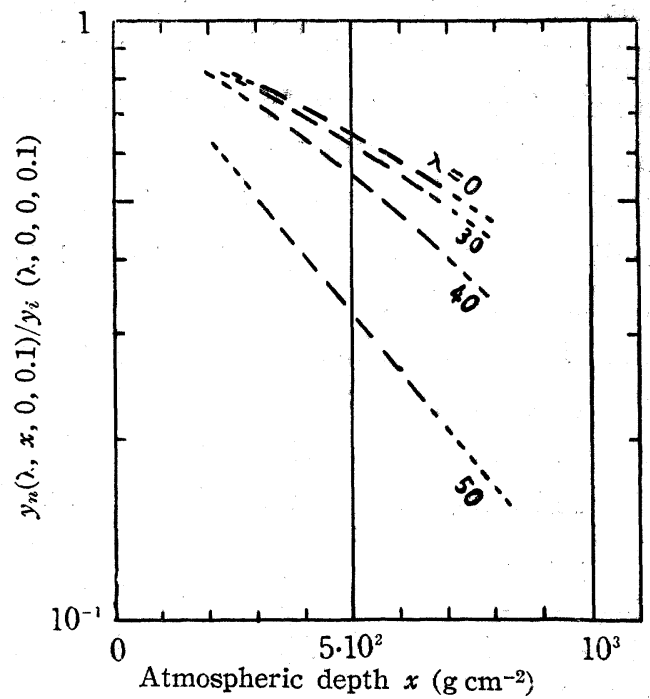

Fig. 12 Altitude dependence of $y$ of the neutron component by the variation of the cut-off energy.

\section{§ 4. Comparison with Observations and Discussion}

Based on the calculations in $\S 2$ and $\S 3$, we consider which variation of either the electric field or the cut-off energy is best suited to explain the world-wide variation of cosmic ray intensity (not dependent on local time) which has three characteristics as cited in the Introduction.

[I] The variation is world-wide.

In case of the electric field:--This can be explained if only the potential on the earth varies. Moreover, as pointed out by H.V. Neher, ${ }^{(3)}$ the fluctuation in intensity must be the same all over the knee of the latitude curve of intensity. This phenomenon can be also explained, independent of the formation of the knee, as shown in Figs. 5 and 6. 
In case of the cut-off energy:--At low latitudes, the changes occur without any condition if the state of the static electric and magnetic field varies, but some difficulty arises in high latitudes. As pointed out by various authors, ${ }^{(1),(0),(19)}$ the change cannot be produced over the knee by the variation of the cut-off energy. They are right if the static electric and magnetic field is symmetrical to the earth's magnetic dipole axis, ${ }^{(3)}$ since such a field, however large, does not produce any variation of cut-off energy near the magnetic poles and then does not satisfy the condition that the variation must be the same all over the knee. On the contrary, if we consider the field not to be symmetrical to the earth's magnetic dipole axis, such a field has the possiblity of satisfying the above mentioned condition. The reason is that this field, a simple example of which is the solar magnetic field, has an agency to cut off the low energy region of the primary spectrum, ${ }^{(20)}$ so that, if such a field is produced or changed by some mechanism, the general cut-off energy $E_{g}$ is changed and then the intensity variation is produced and is the same all over the knee. But a difficulty arises even in such a case; at the normal state, the knee at low altitude is caused by the atmospheric absorption and not by the general cut-off energy $E_{g}$, so that, in expectation of the intensity decrease over the knee at such a low altitude, the general cut-off energy must become greater than the cut-off energy $E_{\lambda}$ at the latitude where the knee at low altitude begins. If such a variation of $E_{g}$ is produced, the latitudes, at which the knees at various altitudes begin, must become constant. Such a phenomenon has not yet been reported. Moreover, we cannot expected any intensity increase from the normal state over the knee at low altitude by such a field, since the decrease of $E_{g}$ gives no influence upon the intensity over the knee at low altitude.

As discussed above, it seems to be difficult to explain the phenomenon [I] by the variation of cut-off energy, although there is some slight possibility of doing so.

[II] The latitude dependence of the variation

Up to date, not much has yet been done about the latitude effect of the worldwide variation at sea level. This is due to the following difficulties: (1) The observation stations are not located at the same altitude, (2) each station is located at different longitude and (3) the type of the cosmic ray meter is different at each station. As we know nothing about these corrections for the variation, the true latitude dependence of $y$ at sea level cannot be obtained. In these corrections, (1) gives the most important influence upon the latitude dependence of $y$.

In case of the electric field:-Keeping the above in mind, we discuss the latitude dependence of $y$, based on the observed results obtained by several authors. ${ }^{(4),(6) \text {, }}$ (21).(22) The relative values of $y_{i}$ at each station, normalized to that at Cheltenham, are shown in Table II. As seen from this table, at some stations, there are some differences between the values obtained by these authors. For example, the value at Teoloyucan obtained by S.E. Forbush is exceedingly larger than that obtained by S. Yoshida and Y. Kamiya, and another example is that the value at Freiburg is extremely small compared to the values at other stations which are located at the 
same altitude and nearly the same latitude as Freiburg. On the other hand, it is a remarkable point that at Huancayo there is quite a good agreement between them. To compare these data with the theoretical latitude curve of $y_{i}$ in Fig. 5 , it is necessary to make the altitude correction to the values at several stations (i.e. Huancayo, Teoloyucan, Canberra and Hafelekar).

Table II Relative value of $y_{i}$ at each station normalized to that at Cheltenham and not correted the altitude effect.

\begin{tabular}{|c|c|c|c|c|c|c|}
\hline \multirow{2}{*}{ No. Station } & \multirow{2}{*}{$\begin{array}{l}\text { Geomagn. } \\
\text { Latitude }\end{array}$} & \multirow{2}{*}{$\begin{array}{l}\text { Height above } \\
\text { sea level (m) }\end{array}$} & \multicolumn{4}{|c|}{ Relative value of $y_{i}$ obtained by } \\
\hline & & & $\begin{array}{l}\text { S. Yoshida(21) } \\
\text { Y. Kamiya }\end{array}$ & M. Wada(22)* & $\begin{array}{l}\text { S.E. } \\
\text { Forbush(4) }\end{array}$ & $\begin{array}{c}\text { W.H. } \\
\text { Fonger( }^{(6)} \text { \& }\end{array}$ \\
\hline 1 Godhavn & $80^{\circ} \mathrm{N}$ & & $1.12 \pm .13$ & 0.97 & & \\
\hline 2 Bergen & $61 \mathrm{~N}$ & & 1.02 & & & \\
\hline 3 Manchester & $57 \mathrm{~N}$ & & $1.06 \pm .07$ & & & \\
\hline 4 Boston & $54 \mathrm{~N}$ & & 0.97 & & & \\
\hline 5 Cheltenham & $50 \mathrm{~N}$ & & 1.00 & 1.00 & 1.00 & 1.00 \\
\hline 6 Christchurch & $49 \mathrm{~S}$ & & \multirow{2}{*}{$0.95 \pm .21$} & & 0.95 & \\
\hline 7 Friedrichshafen & $49 \mathrm{~N}$ & & & & & \\
\hline 8 Freiburg & $49 \mathrm{~N}$ & & & & & 0.77 \\
\hline 9 Hafelekar & $48 \mathrm{~N}$ & 2300 & $1.33 \pm .03$ & & 1.43 & \\
\hline 10 Canberra & $45 \mathrm{~S}$ & 800 & $0.94 \pm .15$ & & & \\
\hline 11 Teoloyucan & $30 \mathrm{~N}$ & 2285 & $1.18 \pm .08$ & & 1.42 & \\
\hline 12 Tokyo & $25 \mathrm{~N}$ & . & $0.85 \pm .05$ & 0.93 & & \\
\hline 13 East China Sea & $22 \mathrm{~N}$ & & 0.87 & & & \\
\hline I4 Huancayo & $1 \mathrm{~S}$ & 3350 & $0.89 \pm .07$ & 0.92 & 0.90 & 0.91 \\
\hline
\end{tabular}

* The values in this column were obtained from the data of cosmic ray storms.

$\S$ The values in this column were obtained from the data of 27 -day variations.

Let $c_{j}\left(\lambda, x^{\prime}-x, \Delta E, 0\right)$ denote the altitude correction factor to alter $y_{j}$ at $x$ to that at $x^{\prime}$, and the following relation holds

$$
y_{i}(\lambda, 1030, \Delta E, 0)=c_{j}(\lambda, 1030-x, \Delta E, 0) y_{j}(\lambda, x, \Delta E, 0) . \quad(j=i \text { or } n) \cdots(10)
$$

$c_{j}$ is obtained theoretically by using the altitude curve of $y_{j}$ as shown in Figs. 7 and 8 , and is listed in the 4 th and 5th columns of Table III for each station in case $j=i$ and $\Delta E=0.1 B e v$.

Using the altitude correction factor $c_{i}{ }^{I}$, we can obtain the experimental relative value of $y_{i}$ at sea level. The results are shown in Fig. 13 together with the latitude curve of $y_{i}{ }^{I}(\lambda, 1030,0.1,0)$ obtained theoretically. As seen from this figure, there is a fairly good agreement between the observations and the theoretical curve, although the 
Table III Altitude correction factor $c_{i}(\lambda, 1030-x, 0.1,0)$

\begin{tabular}{|l|c|c|c|c|c|}
\hline \multicolumn{1}{|c|}{ Station } & $\begin{array}{c}\text { Geomagn. } \\
\text { Latitude }\end{array}$ & $\begin{array}{c}\text { Height above } \\
\text { sea level }(\mathrm{m})\end{array}$ & $c_{i}{ }^{*}$ & $c_{i}{ }^{I I}$ & Observed value of $c_{i}{ }^{\text {(21) }}$ \\
\hline Boulder & $49^{\circ} \mathrm{N}$ & 1600 & 0.77 & 0.83 & \\
\hline Climax & $48 \mathrm{~N}$ & 3500 & 0.60 & 0.70 & \\
\hline Hafelekar & $48 \mathrm{~N}$ & 2300 & 0.69 & 0.77 & 0.71 (Cheltenham/Hafelekar) \\
\hline Bagnéres & $46 \mathrm{~N}$ & 550 & 0.94 & 0.95 & \\
\hline Canberra & $45 \mathrm{~S}$ & 800 & 0.87 & 0.92 & 1.06 (Cheltenham/Canberra) \\
\hline Teoloyucan & $30 \mathrm{~N}$ & 2285 & 0.77 & 0.87 & 0.725 (Tokyo/Teoloyucan) \\
\hline Huancayo & $1 \mathrm{~S}$ & 3350 & 0.80 & 0.96 & \\
\hline
\end{tabular}

* $I$ and $I I$ denote the correction factors obtained by using the value $y_{i}{ }^{I}(\lambda, 1030,0.1,0)$ and $y_{i}{ }^{I L}(\lambda, 1030,0.1,0)$ respectively, as shown in Figs. 5 and 7.

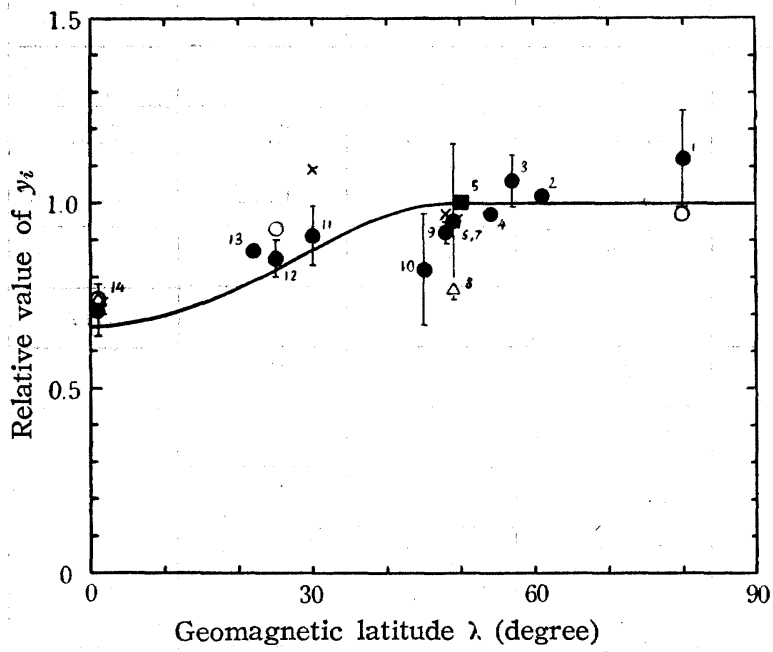

Fig. 13 Latitude dependence of $y_{i}$ at sea level, normalized to that at Cheltenham. Numbers denote Stations in Table III.

- S. Yoshida and Y. Kamiya

M. Wada

S.E. Forbush

W.H. Fonger

Normalization point (Cheltenham)

differences between them are large at some stations (for example, Freiburg and Canberra). The experimental latitude dependence of $y_{i}$ at sea level, corrected by $c_{i}{ }^{I I}$, also has a fairly good agreement with the theoretical one, but is not shown here. As pointed out previously, there is quite a good agreement between the experimental values of $y_{i}$ at Huancayo, obtained by various authors. The value without the altitude correction is equal to $0.89 \pm 0.07$ which was obtained by S. Yoshida and Y. Kamiya. On the other hand, the theoretical value is obtained from Figs. 5 and 7 and equal to 0.82 in case $y_{i}{ }^{I}$ is adopted and 0.78 in case $y_{i}{ }^{I I}$ is adopted. The theoretical value in the former case is better than that in the latter, and is in accordance with the observed value within the experimental error. From Figs. 5 and 6, we see that the latitude dependence of $y$ at high altitude satisfies qualitatively the condition that the changes must increase with the increase of latitude.

S. Yoshida and Y. Kamiya ${ }^{(21)}$ obtained the altitude correction factors at several stations from the observation by comparing the values of $y_{i}$ at two stations which were located at different altitudes respectively and nearly the same latitude each other. The results are tabulated in the last column of Table III. We see that there 
are fairly good agreements between the theoretical and experimental altitude correction factors except that at Canberra. Although it is difficult to interpret that the experimental value at Canberra becomes larger than unity, this might be due to the scarcity of observations or the difference of cosmic ray meter compared with that at other stations.

As seen from the above comparisons of the theoretical values with the observations, the latitude dependence of the world-wide variation is explained by the variation of the static electric field.

In case of the cut-off energy:--As pointed out in $\S 3$, the latitude dependence of the variation cannot be obtained unless the state of the electro-magnetic field is determined, so that the comparison of the theoretical curve with the observation cannot be done.

[III] The altitude dependence of the variation

Recently, the world-wide variations of the ionizing and neutron components at various altitudes have been observed by various authors. ${ }^{(3),(23),(24)}$ H.V. Neher pointed out that the ratio of the variation at high altitude to the variation at sea level is always constant. Such ratios obtained experimentally are reproduced in the 3rd column of Table IV. The theoretical ratios expected from the variation of the static electric field are in the 4 th column where I and II represent the values obtained by using $y_{i}{ }^{I}(\lambda, 1030,0.1,0)$ and $y_{i}{ }^{I I}(\lambda, 1030,0.1,0)$, respectively. In case of the variation of cut-off energy, it is possible to obtain the ratio of the variation at the same

Table IV Altitude dependence of $y$

\begin{tabular}{|c|c|c|c|c|c|c|}
\hline \multirow[t]{2}{*}{ No. } & \multirow[t]{2}{*}{$y_{j}(\lambda, x): y_{k}(\lambda, x)$} & \multirow[t]{2}{*}{$\begin{array}{l}\text { Observed } \\
\text { value }\end{array}$} & \multicolumn{2}{|c|}{$\begin{array}{c}\text { Value expected from } \\
\text { electric field }\end{array}$} & \multirow{2}{*}{$\begin{array}{l}\text { Value expected } \\
\text { from cut-off } \\
\text { energy }\end{array}$} & \multirow[t]{2}{*}{ Remarks } \\
\hline & & & I & II & & \\
\hline 1 & $\begin{array}{c}y_{i}(56,50): y_{i i}(56,1030) \\
\text { Bismarck }\end{array}$ & $7: 1$ & $7: 1$ & $5.6: 1$ & & (3) \\
\hline 2 & $\begin{array}{ll}y_{i}(45,50): & y_{i}(1,700) \\
\text { Chicago } & \text { Huancayo }\end{array}$ & $6: 1$ & $4.6: 1$ & $3.6: 1$ & $50: 1$ & (3) \\
\hline 3 & $\begin{array}{c}y_{i}(43,50): y_{i}(41,880) \\
\text { Ft. Worth } \\
\text { Texas }\end{array}$ & $4: 1$ & $2.6: 1$ & $2.3: 1$ & $12: 1$ & $\begin{array}{l}\text { This ratio was } \\
\text { obtained at the } \\
\text { time of the } \\
\text { magnetic storm }\end{array}$ \\
\hline 4 & $\begin{array}{ll}y_{n}(48,680): & y_{i}(50,1030) \\
\text { Climax } & \text { Cheltenham }\end{array}$ & $4.0: 1$ & $3.9: 1$ & $3.1: 1$ & $50: 1$ & $(6)$ \\
\hline 5 & $\begin{array}{ll}y_{n}(48,680): & y_{i}(49,1000) \\
\text { Climax } & \text { Freiburg }\end{array}$ & $5.5: 1$ & & & & (6) \\
\hline 6 & $\begin{array}{ll}y_{n}(48,680): & y_{i}(1,700) \\
\text { Climax } & \text { Huancayo }\end{array}$ & $4.5: 1$ & $4.8: 1$ & $4.0: 1$ & $50: 1$ & (6) \\
\hline
\end{tabular}

Numbers in the remarks column denote those of References quoted.

latitude, but not possible to obtain the ratio if stations are located at different latitudes, because, as stated in $\S 3$, the latitude curve of $y$ cannot be obtained unless the state of the electro-magnetic field is determined. But, if it is assumed that the intensity variation caused by the variation of cut-off energy shows no latitude effect 
at ground level as the world-wide variation actually observed at the ground shows no remarkable latitude effect, it is possible to obtain the ratio between two stations which are located at different latitudes. The results are shown in the 5th column of Table IV. As stated above in [I], the variation of cut-off energy could possibly produce the intensity variation at high latitude, but here no such ratio is obtained.

As seen from this Table, the observed ratios show better agreements with the theoretical ones expected from the variation of electric field than that expected from the variation of cut-off energy. Moreover, in case of the electric field, the values in case I are better than those in case II. But, in point of detail, there are some discrepancies observable between the theoretical and experimental values as shown in the 2nd and 3rd rows of Table IV. These discrepancies seem to increase with the decrease of latitude:

In the 4 th, 5th and 6 th rows, the ratios between the neutron variation at Climax and the ionizing variations at several stations are shown. The observed ratio adopted by W.H. Fonger in order to compare with his theoretical calculation is that cited in the 5th row. But, as pointed out previously and seen from Table II and Fig. 13, the variation of the ionizing component at Freiburg is very small, compared with the variations at other stations which are located at the same latitude as Freiburg, so that the ratio in the 5th row is much larger than that obtained by using the variation at the other stations. Then, in order to compare with the theoretical value, it is more reasonable to adopt the values given in the 4 th and 6 th rows, than to adopt the value given in the 5th row. As for the ratio of the neutron component at Climax to the ionizing component at Cheltenham or Huancayo, there is quite a good agreement between the experimental and theoretical values in case I.

As stated in $\S 2$, the variation of the neutron component must be independent on altitude in the atmospheric depth greater than $600 \mathrm{~g} \mathrm{~cm}^{-2}$, if the observation of the neutron intensity made by J.A. Simpson et al. ${ }^{(18)}$ is correct. Therefore, to confirm this fact, it is most desirable to make the comparisons between the variation of the neutron component at Climax and those at the other stations.

As seen from the above various considerations and discussions, it is possible to explain all the phenomena of the world-wide variation by the variation of the earth's potential, although in point of detail there are some discrepancies observable between the experimental and theoretical values. On the contrary, the variation of cut-off energy cannot explain the altitude dependence even though it may possibly satisfy the phenomenon [I].

[IV] The large variation of cosmic ray intensity

The large decrease of $6 \%$ or more in cosmic ray intensity at sea level are often observable at the time of the cosmic ray storm. ${ }^{(25)}$ In such cases, the altitude and latitude dependences of the variation calculated in $\S 2$ do not hold, because, as pointed out in $\S 1$, they depend on the magnitude of the variation of earth's potential.(11) We must therefore calculate them when the variation of earth's potential is large. Figs. 14 and 15 represent the latitude dependences of $y$ and Figs. 16 and 17, the altitude 
dependences of $y$, in case $\Delta E=1 \mathrm{Bev}$. The method of the normalization of the curves in Figs. 16 and 17 is the same as that adopted in Figs. 7 and 8. From these figures, we see that the altitude and latitude dependences become smaller if $\Delta E$ becomes larger. Moreover, to make this feature more clear, Figs. 18 and 19 are shown, representing the variations of the ionizing and neutron components at various altitudes
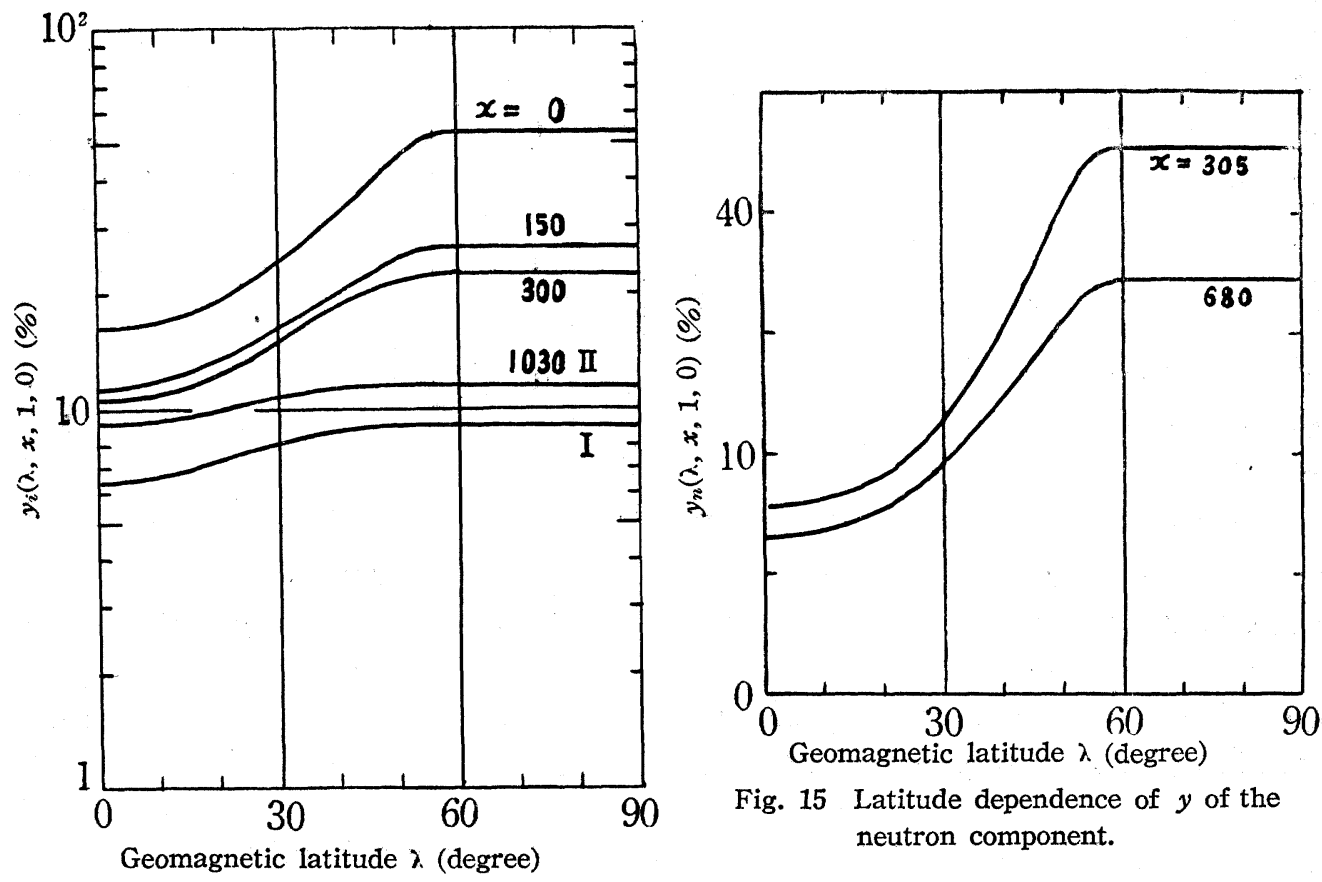

Fig. 15 Latitude dependence of $y$ of the neutron component.

Fig. 14 Latitude dependence of $y$ of the ionizing component.

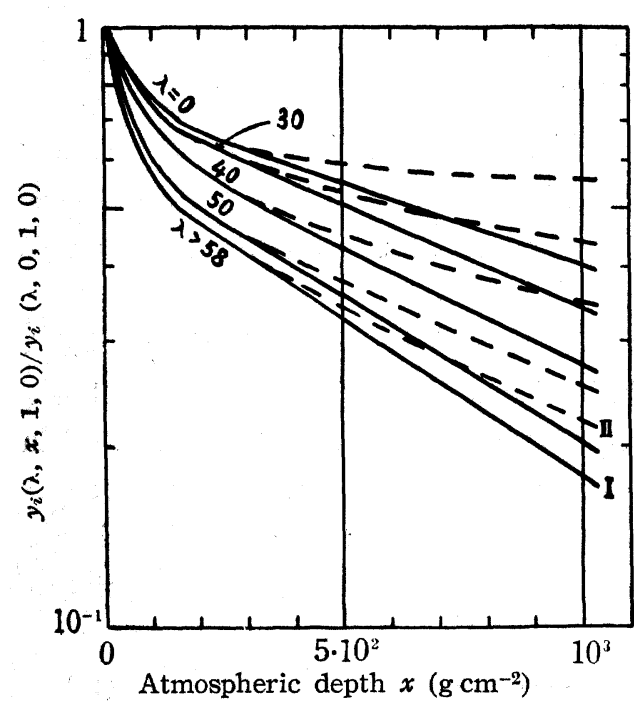

Fig. 16 Altitude dependence of $y$ of the ionizing component.

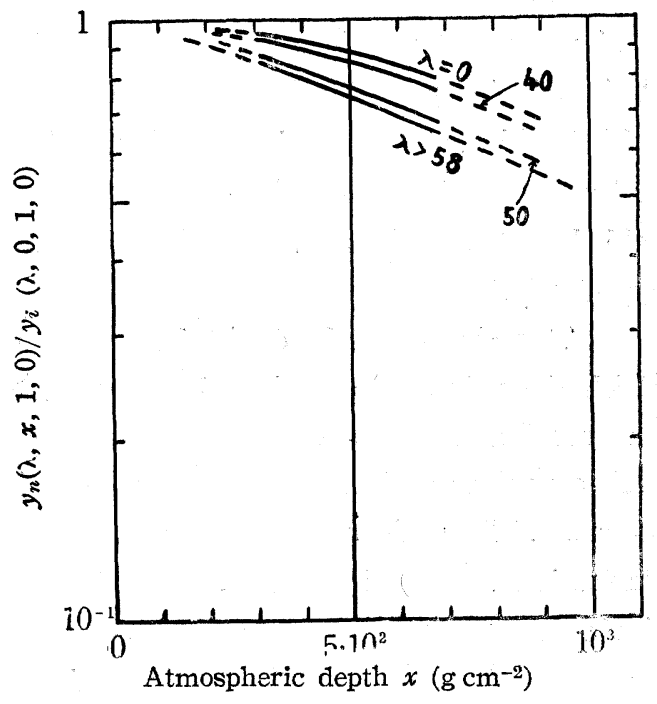

Fig. 17 Altitude dependence of $y$ of the neutron component. 
as a function of $\Delta E$, in case $\lambda \geq 58^{\circ}$. As seen from these figures, the dependence of $y$ on $\Delta E$ becomes smaller with the increase of $x$, and it can be concluded that $y$ is approximately proportional to $\Delta E$ at low altitudes and latitudes. But, at high altitudes and latitudes, it depends exceedingly on the magnitude of $\Delta E$. Then it is necessary

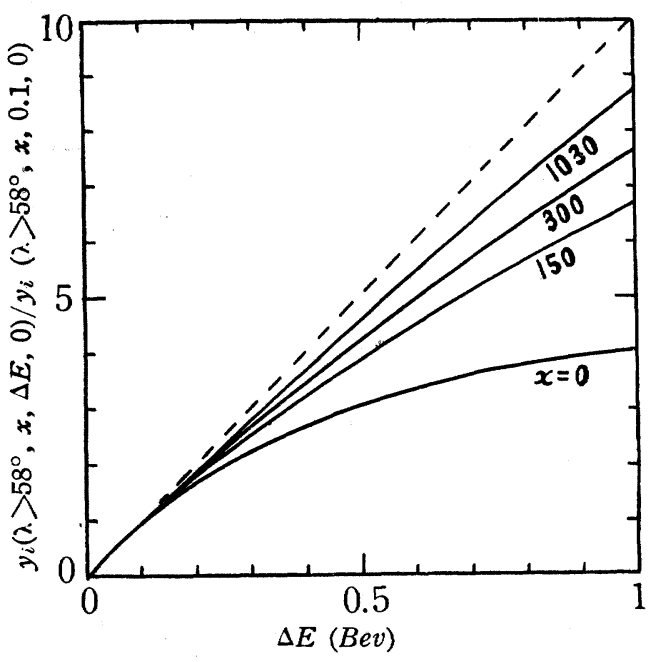

Fig. $18 \Delta E$ dependence of $y$ of the ionizing component.

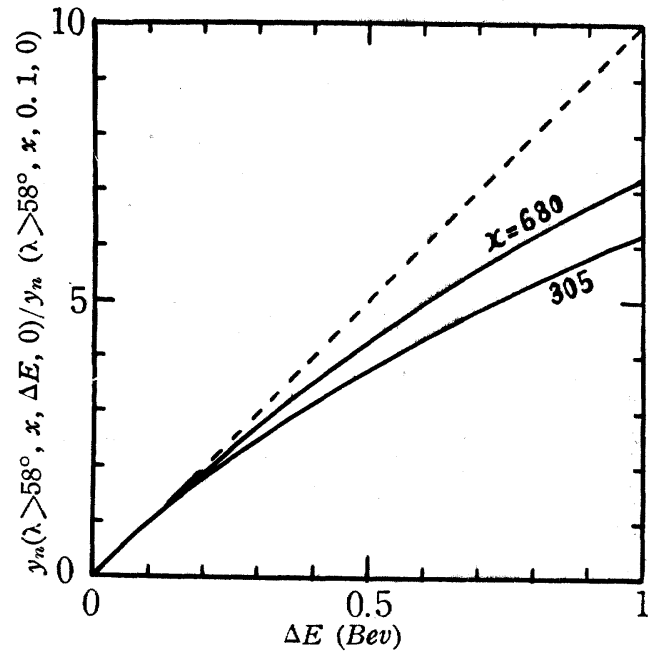

Fig. $19 \Delta E$ dependence of $y$ of the neutron component.

to consider such an influence upon the variation in order to compare the experimental altitude dependence of $y$ with the theoretical one.

\section{§ 5. The Diurnal Variation of Cosmic Ray Intensity}

The diurnal variation of cosmic ray intensity is also a world-wide phenomenon at sea level, whose difference from the world-wide variation discussed in the previous sections is that the former depends on local time. Here, an examination is to be made of the possibility that the diurnal variation can be explained by an electric field ${ }^{(26)}$ although the origin of such an electric field cannot be pointed out at present; and a qualitative comparison of the diurnal variation expected from such an electric field hypothesis is to be made with that expected from the static magnetic field hypothesis. ${ }^{(7)}$

The electric field derived from a potential can no longer explain the variation which depends on local time, because the variation is produced simultaneously all over the world as long as the electric field is derived from a potential.(2r) Then, to explain such a variation, it is necessary to consider an electric field not derived from a potential. In such a general electro-magnetic field as well as the static electric and magnetic field, the intensity of cosmic rays, whose momentum are $P$ at any point, is also expressed by

$$
i=P^{2} D
$$

where

$$
D=\frac{\delta N}{\delta v}, \text { with } \delta v=\delta x \delta y \delta z \delta P_{x} \delta P_{y} \delta P_{z}, \cdots \cdots \cdots
$$


and $\delta N$ is the number of particles in the volume $\delta v$. Even in such a general field, the extended Liouville's theorem ${ }^{(28),(29)}$ holds and then $D$ in Eq. (11) is constant as well as in the static electric and magnetic field. ${ }^{(30)}$ But, the difference in both cases is that, in the latter case, $P$ is determined if only the electric potential at any point is known; in the former case, on the contrary, $P$ cannot be determined unless the trajectory of charged particle can be obtained in the general electro-magnetic field. Keeping this point in mind and assuming that the primary particles consist of protons only, we are able to obtain also the vertical cosmic ray intensity at geomagnetic latitude $\lambda^{\circ}$ and atmospheric depth $x \mathrm{~g} \mathrm{~cm}^{-2}$ in the general field, using the method similar to that adopted in the static field.(1)

$$
\begin{aligned}
N\left\{\lambda, x, \Delta E(E, \lambda, t), \delta E_{\lambda}\right\}=\int_{E_{\lambda}+\delta E_{\lambda}}^{\infty} m(E, x)[1-L\{E+\Delta E(E, \lambda, t), \Delta E(E, \lambda, t)\}] & \\
& \times i\{E+\Delta E(E, \lambda, t)\} d E, \cdots(13)
\end{aligned}
$$

where $N, \delta E_{\lambda}, m, L$ and $i$ are the same notations as those in Eq. (3), and $\Delta E(E, \lambda, t)$ is the energy loss which the primary particle, having its kinetic energy $E$ at $\lambda^{\circ}$ on the earth, undergoes during its passage through the electro-magnetic field, and is a function of $E, \lambda$ and local time $t$, since $\Delta E$ depends upon the particle's trajectory which can be determined by parameters $E, \lambda$ and $t$. Although it is meaningless to try to determine whether such a variation is caused by the electric field or the magnetic field, we designate such a variation for the variation caused by the general electric field in distinction from the variation caused by the static electric field or by the static magnetic field, since the agency of the electro-magnetic field upon the intensity variation is similar to that of the static electric field as seen from Eqs. (8) and (13).

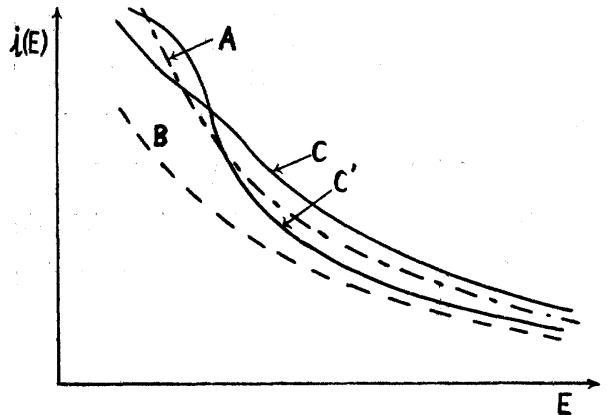

Fig. 20 Variation of primary intensity spectrum produced by the electric field.

1. Curve A represents the spectrum at the normal state.

2. Curve $B$ represents the variation of the spectrum from the normal state produced by the static electric field.

3. Curve $\mathrm{C}$ and $\mathrm{C}^{\prime}$ represent the variations of the spectrum produced by the general electric field at the times $t$ and $t^{\prime}$ respectively.
Fig. 20 is a schematic representation of deformations of the primary spectrum which will be caused by the general electric field. The distinction between the intensity variation caused by the static electric field and that caused by the general electric field is as follows: In the former case, the relative contribution of the primary particles, each having a different energy, to the intensity variation is uniquely determined (see Eq. (8) and Curve B in Fig. 20). In the latter case, on the contrary, the relative contribution varies with local time (see Eq. (13) and Curves $\mathrm{C}$ and $\mathrm{C}^{\prime}$ in Fig. 20). Then, the altitude dependence of diurnal variation is different from that of the world-wide variation expected from the static electric field and cannot be obtained theoretically unless 
the state of the electo-magnetic field is determined.

Comparing the diurnal variation of neutron component at Climax with that of ionizing component at Freiburg, W.H. Fonger ${ }^{(6)}$ showed that the ratio between the amplitudes of variation at the two stations is nearly equal to the ratio of the world: wide variation (not dependent on local time) expected from the static electric field hypothesis (here-after referred to as Phenomenon b). If we substitute the altitude dependence curve obtained from the static electric field for the true altitude depend. ence curve of diurnal variation as a rough approximation because of the existence of Phenomenon $b$, the amplitude of diurnal variation of the ionizing component at high latitude will be expected to be about 10 times that of sea level at the top of the atmosphere and about 7 times at $x=50 \mathrm{~g} \mathrm{~cm}^{-2}$.

The static electric and magnetic field also has the possibiblity of producing the diurnal variation, if the field is not symmetrical to the earth's magnetic dipole axis. There is the solar magnetic field theory adovocated by M.S. Vallarta, ${ }^{(7)}$ as the repre. sentative example. The altitude dependence of diurnal variation expected from this theory is smaller than that expected from the variation of cut-off energy, because, as pointed out in $\S 1$, the diurnal variation is caused by the variation of primary spect. rum at intermediate energy region and not by the variation of cut-off energy. The distinction between the general electric field hypothesis and the solar magnetic field theory is as follows: The altitude dependence of diurnal variation expected from the former is smaller than that expected from the latter, ${ }^{(31)}$ since, in the latter case, only the primary particles in the low energy region are influenced; in the former case, on the contrary, all the particles in all of the energy region are influenced.

As above, the diurnal variation could possibly be explained by the electric field not derived from potential, even though it cannot indicate the mechanism producing such an electric field. Recently, the diurnal variation at high altitude was observed by various authors ${ }^{(32)}$ in order to confirm the existence of solar magnetic field. But, none of them has reported the ratio between the amplitude of diurnal variation at high altitude and that at sea level. It is most important to examine the altitude dependence from the experimental and theoretical points of view in order to determine the cause of the diurnal variation.

\section{Concluding Remarks}

It is concluded that the world-wide variation of cosmic ray intensity not depend. ent on local time can be explained by the variation in the distribution of the primary cosmic ray intensity spectrum which is caused by the variation of the static electric field, but not explained by the variation of cut-off energy which is caused by the variation of the electro-magnetic field.

The diurnal variation of cosmic ray intensity could possibly be explained by an electric field not derived from potential. The existence of Phenomenon $b$ seems to be favourable to the general electric field hypothesis. 


\section{Acknowledgements}

In conclusion, the writer wishes to express his hearty thanks to Prof. M. Hasegawa, for his constant interest in and encouragement of the present research, and to Profs. Y. Sekido and T. Nagata as well as Mr. S. Hayakawa for their many valuable criticisms and discussions in relation to this work. Many thanks are due to Profs. M.S. Vallarta and J.A. Wheeler for their valuable discussions relative to this work on their visit to Japan.

\section{Appendix I The Variation of Cut-Off Energy by the Static Electric and Magnetic Field}

The relativisitic equation of the charged particle's motion in the electro-magnetic field is

$$
\frac{d}{d t}(m v)=e E+\frac{e}{c}[v H]
$$

General solution of Eq. (A,1) cannot be obtained analytically even in case of the motion in the earth's dipole magnetic field. Then it is extremely difficult to solve Eq. $(A, 1)$ in case the static electric and magnetic field surrounding the earth exists in addition to the earth's magnetic field. But, in one special case, it is possible to obtain

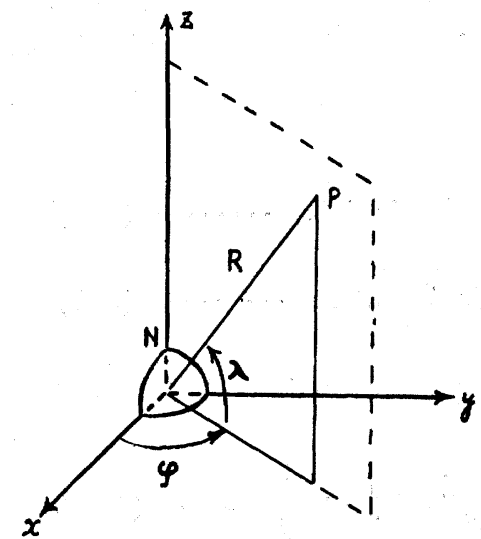

Fig. 21 Coordinate system the Störmer cone ${ }^{(33)}$ and then to obtain the variation of cut-off energy caused by the static electric and magnetic field. As the coordinate system, here are the spherical coordinates $(R, \lambda, \varphi)$ adopted, as shown in Fig. 21, and the $z$-axis coinciding with the earth's magnetic dipole axis and directed towards the North Pole.

If the static electric and magnetic field surrounding the earth is symmetrical to $z$-axis, the following conditions hold;

$$
\boldsymbol{E}_{\varphi}=0 \text { and } \boldsymbol{H}_{\varphi}=0 . \cdots \cdots(\mathrm{A}, 2)
$$

Then, in such a case, the $\varphi$-component of Eq. $(A, 1)$ becomes

$$
\frac{d}{d t}(m v)_{\varphi}=\frac{e}{c}[v H]_{\varphi}
$$

In Eqs. $(\mathrm{A}, 1)$ and $(\mathrm{A}, 3)$, the mass $m$ is not constant because of the existence of the electric field. But, quite similar to the case that only the static magnetic field exists, Eq. $(A, 3)$ can be integrated, and the solution is

$$
m(R \cos \lambda)^{2} \frac{d \varphi}{d t}=\frac{e}{c}\left\{-(R \cos \lambda) A_{\varphi}+\Gamma\right\}
$$


where $\Gamma$ is an integral constant, and $\boldsymbol{A}_{\varphi}$ is the $\varphi$-component of vector potential and in this case is given by

$$
\boldsymbol{A}_{\varphi}=-\frac{M \cos \lambda}{R^{2}}-\boldsymbol{A}_{\varphi}^{0}
$$

The 1st term on the right hand side of Eq. $(A, 5)$ is due to the earth's dipole magnetic field, $\boldsymbol{M}$ denoting the magnetic dipole moment, and $-\boldsymbol{A}_{\varphi}^{0}$ to the outer field. Let $\theta$ denote the angle between the particle's orbit and the meridian plane, and the next relation is obtained by using Eq. $(A, 4)$.

$$
\sin \theta=\frac{R \cos \lambda}{v} \cdot \frac{d \varphi}{d t}=\frac{e}{m v c}\left\{\frac{M \cos \lambda}{R^{2}}+\boldsymbol{A}_{\varphi}^{0}(R, \lambda)+\frac{I}{R \cos \lambda}\right\} \cdot \cdots \cdots(\mathrm{A}, 6)
$$

Then, the motion must satisfy the following condition:

$$
\left|\frac{e}{m v c}\left\{\frac{M \cos \lambda}{R^{2}}+\boldsymbol{A}_{\varphi}^{0}(R, \lambda)+\frac{\Gamma}{R \cos \lambda}\right\}\right| \leqq 1 .
$$

Next, we consider the extended Störmer transformation ${ }^{(33),(34)}$ as follows,

and

$$
l=\sqrt{\frac{|\boldsymbol{e}| \bar{M}}{(m v c)_{0}}} \quad \text { and } \quad R=l r, \text { etc., }
$$

$$
(m v c)=(m v c)_{0}\left[1-L\left\{E_{0}, e \delta \phi(R, \lambda)\right\}\right]^{\frac{1}{2}}, \cdots \cdots \cdots \cdots
$$

where $L$ is Liouville's effect and given by Eq. (4), $E$ is the kinetic energy corresponding to the value of $m v c$, and subscript odenotes the value on the earth. $\delta \phi(R, \lambda)$ is the difference in electric potential relative to the earth's potential at any point $(R, \lambda)$. Appling these transformations to Eqs. $(A, 6)$ and $(A, 7)$, the following relations can be obtained;

$$
\begin{gathered}
\sin \theta=\frac{1}{(1-L)^{1 / 2}}\left\{\frac{\cos \lambda}{r^{2}}-\frac{2 \gamma_{1}}{r \cos \lambda}+\frac{l^{2}}{M} A_{\varphi}^{0}(l r, \lambda)\right\} \\
\left|\frac{1}{(1-L)^{1 / 2}}\left\{\frac{\cos \lambda}{r^{2}}-\frac{2 \gamma_{1}}{r \cos \lambda}+\frac{l^{2}}{M} A_{\varphi}^{0}(l r, \lambda)\right\}\right| \leq 1
\end{gathered}
$$

where $2 \gamma_{1}$ is the integral constant. From Eq. $(A, 7)^{\prime}$, we can obtain the following relation ;

where

$$
r \cos \lambda\left(\frac{\cos \lambda}{r^{2}}+1+\alpha-\beta\right) \geq 2 \gamma_{1} \geq r \cos \lambda\left(\frac{\cos \lambda}{r^{2}}-1+\alpha+\beta\right)
$$

$$
\left.\begin{array}{l}
\alpha\left(r, \lambda, r_{0}\right)=\frac{l^{2}}{M} \boldsymbol{A}_{\varphi}^{0}(l r, \lambda), \\
\beta\left(r, \lambda, r_{0}\right)=1-\left[1-L\left\{E_{0}, e \delta \phi(l r, \lambda)\right\}\right]^{\frac{1}{2}} .
\end{array}\right\}
$$

$\alpha$ and $\beta$ are the terms due to the outer magnetic and electric fields respectively, and are functions of $r, \lambda$, and $r_{0}$ (which is the particle's energy in Störmer unit ${ }^{(33)}$ corresponding to $E_{0}$ or $\left.(m v c)_{0}\right)$. Eq. $(\mathrm{A}, 10)$ depends upon the particle's energy, different from the case where only the earth's magnetic field exists. Hence, the Störmer transformation loses its usefulness in this case. 
As the influence of the outer magnetic field upon the cut-off energy has been examined by various authors, ${ }^{(35)}$ here that of the electric field only is considered. In this case, Eq. $(A, 10)$ becomes as follows,

$$
r \cos \lambda\left(\frac{\cos \lambda}{r^{2}}+1-\beta\right) \geq 2 \gamma_{1} \geq r \cos \lambda\left(\frac{\cos \lambda}{r^{2}}-1+\beta\right) .
$$

Fig. 22 is a schematic representation of Eq. $(A, 12)$ in case $\lambda=0$. The full lines

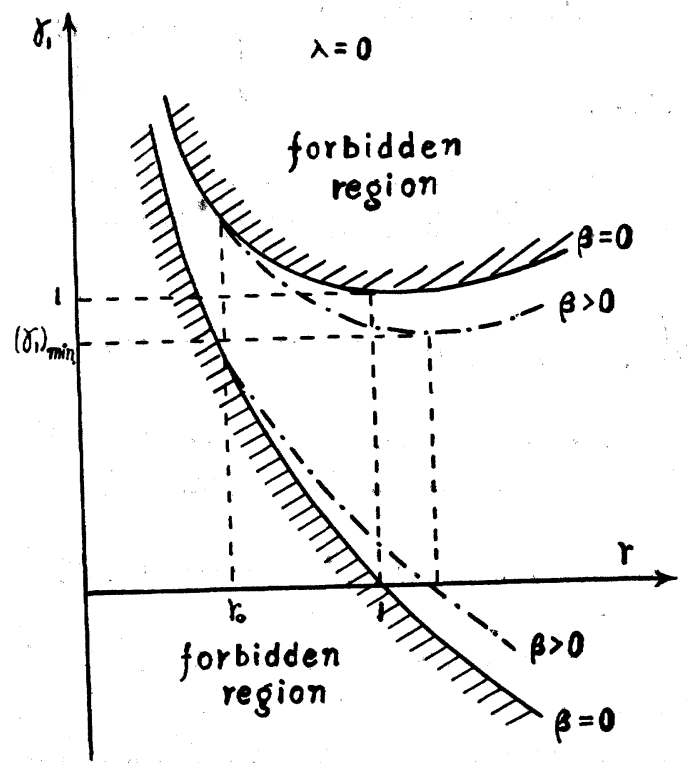

Fig. $22 \quad \gamma_{1}-r$ diagram represent the curves $\gamma_{1}=\frac{r}{2}\left(\frac{1}{r^{2}}+1\right)$ and $r_{1}=\frac{r}{2}\left(\frac{1}{r^{2}}-1\right)$, which equations denote the non-existence of the electric field and are tenable independent of the particle's energy under consideration. When the electric field exists, these curves cease to be independent of the particle's energy. The dotted lines in Fig. 22 represent an example of the deviations from the normal state produced under the influence of the electric field, in case of the particle's energy becoming $r_{0}$ Störmer $\left(r_{0}<1\right)$ on the earth. To examine the variation of cut-off energy in such a case, it is necessary to obtain the minimum value of the following curve;

$$
2 \gamma_{1}=r\left\{\frac{1}{r^{2}}+1-\beta\left(r, 0, r_{0}\right)\right\} \text {. }
$$

Generally, this problem cannot be solved unless the form of $\beta$ is determined. But an analysis can proceed when the disturbance by the electric is small. Under such a condition, $\beta$ in Eq. $(A, 11)$ is approximately given by

$$
\begin{aligned}
\beta\left(r, 0, r_{0}\right) & \approx \beta_{a p .}\left(r, 0, r_{0}\right)=\frac{1}{2} L\left\{E_{0}, e \delta \phi\left(r, 0, r_{0}\right)\right\} \\
& \approx \frac{\left(E_{0}+m_{0} c^{2}\right) \cdot e \delta \phi\left(r, 0, r_{0}\right)}{\left(E_{0}+m_{0} c^{2}\right)^{2}-\left(m_{0} c^{2}\right)^{2}} \ll 1, \quad \ldots
\end{aligned}
$$

where $E_{0}$ is the particle's energy in Bev., corresponding to $r_{0}$ Störmer. The above equation holds if only $E_{0} \gg e \delta \phi\left(r, 0, r_{0}\right)$. As the minimum value of $\gamma_{1}$ in Eq. (A, 13) is 1 at $\gamma=1$ in case $\beta=0$, it can be assumed that the minimum value of $\gamma_{1}$ will be given by

at

$$
\left.\begin{array}{rl}
\left(\gamma_{1}\right)_{m i m_{0}}=1+\Delta \gamma_{1}\left(r_{0}, \beta\right), & \left(\Delta \gamma_{1} \ll 1\right) \\
r & =1+\Delta r\left(r_{0}, \beta\right) . \quad(\Delta r \ll 1)
\end{array}\right\}
$$

Let $\beta$ be further developed in the power series at $\gamma=1$; 


$$
\beta\left(r, 0, r_{0}\right)=\beta\left(1,0, r_{0}\right)+\frac{\beta^{\prime}\left(1,0, r_{0}\right)}{1 !}(r-1)+\frac{\beta^{\prime \prime}\left(1,0, r_{0}\right)}{2 !}(r-1)^{2}+\cdots, \ldots \cdots(A
$$

where $\beta\left(1,0, \gamma_{0}\right)$ is the value at $\gamma=1$ and $\beta^{\prime}\left(1,0, r_{0}\right)$ is that of the 1st derivative of $\beta$ with respect to $r$. Using Eqs. $(A, 13),(A, 15)$ and $(A, 16)$, and neglecting the higher order of $\Delta r$ as small, we can obtain the following relations:

$$
\begin{aligned}
& \Delta \gamma_{1}\left(r_{0}, \beta\right)=-\frac{1}{2}\left[\beta\left(1,0, \gamma_{0}\right)+\frac{\left\{\beta\left(1,0, r_{0}\right)+\beta^{\prime}\left(1,0, r_{0}\right)\right\}^{2}}{2-2 \beta^{\prime}\left(1,0, r_{0}\right)-\beta^{\prime \prime}\left(1,0, r_{0}\right)}\right] \\
& \Delta r\left(r_{0}, \beta\right)=\frac{\beta\left(1,0, r_{0}\right)+\beta^{\prime}\left(1,0, r_{0}\right)}{2-2 \beta^{\prime}\left(1,0, r_{0}\right)-\beta^{\prime \prime}\left(1,0, r_{0}\right)}
\end{aligned}
$$

Next, we examine the variation of cut-off energy produced by such a variation of the minimum value of $\gamma_{1}$. In case only the electric field exists, Eq. (A, 6) becomes as follows,

$$
\sin \theta=\frac{1}{(1-L)^{1 / 2}}\left(\frac{\cos \lambda}{r^{2}}-\frac{2 \gamma_{1}}{r \cos \lambda}\right)
$$

Introducing, into the above, the values $\theta=\theta, r=r_{0}, \gamma_{1}=\left(\gamma_{1}\right)_{m i n}$, and $L=0$ (as the potential difference $\delta \phi$ is zero on the earth), the following relation is obtained,

$$
\cos \lambda_{1}=\frac{1}{2}\left[r_{0}^{2} \sin \theta+V \overline{\left(r_{0}^{2} \sin \theta\right)^{2}+8 r_{0}\left(r_{1}\right)_{m i n .}}\right]:
$$

$\lambda_{1}$, satisfying Eq. $(A, 19)$, denotes the latitude at which $r_{0}$ Störmer, being the particle's energy on the earth, the particle entering into the earth from the given direction $\theta$, exactly becomes the cut-off energy. In case the electric field does not exist, let $\lambda_{0}$ be the latitude at which $r_{0}$ Störmer becomes the cut-off energy, and $\lambda_{0}$ is given by

$$
\cos \lambda_{0}=\frac{1}{2}\left[r_{0}^{2} \sin \theta+\sqrt{\left(r_{0}^{2} \sin \theta\right)^{2}+8 r_{0}}\right]
$$

From Eqs. $(A, 19)$ and $(A, 20)$, we get

where

$$
\left.\begin{array}{l}
\cos \lambda_{1} \approx \cos \lambda_{0}+\eta, \\
\eta=\frac{2 r_{0} \cdot \Delta \gamma_{1}}{\sqrt{\left(r_{0}^{2} \sin \theta\right)^{2}+8 r_{0}}},
\end{array}\right\} \quad \cdots \cdots \cdots \cdots
$$

in which $\Delta \gamma_{1}$ is given by Eq. (A,17). Moreover, in case the electric field does not exist, let $r_{1}$ Störmer be the cut-off energy at the latitude $\lambda_{1}$, we get the following relation, ${ }^{(36)}$

$$
r_{1}=\cos ^{2} \lambda_{1}\left[1+V \overline{\cos ^{3} \lambda_{1} \sin \theta}\right]^{-1}
$$

Introducing Eq. $(A, 21)$ into the above, the following relation holds between $r_{1}$ and $r_{0}$, where

$$
\left.\begin{array}{l}
r_{1} \approx r_{0}(1+\delta), \\
\delta=\left(\frac{2}{\cos \lambda_{0}}-\frac{3}{2} \cdot \frac{r_{0} \sin \theta}{r_{0} \cos \lambda_{0} \sin \theta+1}\right) \eta .
\end{array}\right\}
$$

If the energies $r_{1}$ and $r_{0}$ Störmers in Eq. $(\mathrm{A}, 23)$ are converted into the energies $E_{1}$ and $E_{0}$ in $B \mathbf{e} v$. respectively, Eq. $(\mathrm{A}, 23)$ becomes 
where

$$
E_{1}=E_{0}+\zeta
$$

and

$$
\left.\begin{array}{l}
\zeta=\frac{\left(E_{0}+m_{0} c^{2}\right)^{2}-\left(m_{0} c^{2}\right)^{2}}{E_{0}+m_{0} c^{2}} \cdot(2 \delta), \\
r_{0}=\left(\frac{R_{e}}{e^{2} M^{2}}\right)^{\frac{1}{4}}\left[\left(E_{0}+m_{0} c^{2}\right)^{2}-\left(m_{0} c^{2}\right)^{2}\right]^{\frac{1}{4}},
\end{array}\right\}
$$

in which $R_{e}$ is the earth's radius. From Eqs. $(\mathrm{A}, 21)$ and $(\mathrm{A}, 24)$, we see that the particle's energy $E_{0}$, which is the cut-off energy at $\lambda=\lambda_{0}$ and $\theta=\theta$ in case of the nonexistence of the electric field, becomes the cut-off energy at $\lambda=\lambda_{1}$ and $\theta=\theta$ in case of the existence of the electric field, and that the relation between $E_{0}$ and $E_{1}$ (which is the cut-off energy at $\lambda=\lambda_{1}$ and $\theta=\theta$ in case of the non-existence of the electric field) is given by Eq. (A, 24).

To make the physical meaning of these equations more clear, the simplification of these equations is carried out. In Eq. (A, 17), $\Delta \gamma_{1}$ is a function of $\beta, \beta^{\prime}$ and $\beta^{\prime \prime}$, and then cannot be determined unless the space distribution of the electric potential is known. But, if we assume that the potential does not vary rapidly near the point where $r=1$ and $\lambda=0$ so as to satisfy the following relations,

$$
\beta^{\prime} \leqslant \beta \leqslant 1 \quad \text { and } \quad \beta^{\prime \prime} \leqslant \beta \leqslant 1 \text {, }
$$

and then, neglecting the higher order of $\beta, \beta^{\prime}$ and $\beta^{\prime \prime}$, Eq. $(A, 17)$ becomes as follows,

$$
\Delta \gamma_{1} \approx-\frac{1}{2} \beta\left(1,0, r_{0}\right) \approx-\frac{1}{2} \frac{\left(E_{0}+m_{0} c^{2}\right)}{\left(E_{0}+m_{0} c^{2}\right)^{2}-\left(m_{0} c^{2}\right)^{2}} \cdot e \delta \phi\left(1,0, \gamma_{0}\right) . \cdots(\mathrm{A}, 25)
$$

Using the above equation, in case of the vertical incidence $(\theta=0)$, Eq. $(A, 24)$ becomes as follows,

$$
E_{1}=E_{0}-e \delta \phi\left(1,0, r_{0}\right) . \quad B e v .
$$

As seen from the above, the variation of the cut-off energy produced by the static electric field is approximately determined only by the earth's potential, and is $-e \delta \phi\left(1,0, r_{0}\right)$ in case of the vertical incidence. When the potential at the point where $r=1$ and $\lambda=0$ is higher than that of the earth $(\delta \phi>0)$, the cut-off energy becomes larger than that of the normal state and then, as discussed in $\S 3$, the cosmic ray intensity is decreased by such a variation. But, as seen from Fig. 9, such a decrease is exceedingly small even in the high atmosphere if $e \delta \phi \leqslant 10^{-2} B e v$. As pointed out by H.V. Neher, ${ }^{(3)}$ there exists the general cut-off energy $E_{g}(=0.8 B e v$. for proton) which corresponds to about 0.16 Störmer. The distance from the earth's center to the point where $r=1$ becomes nearly 6 times the earth's radius even if we consider the lowest energy 0.16 Störmer. Therefore, if the following relation is satisfied over the region less than 6 times the earth's radius,

$$
e \delta \phi \leqslant 10^{-2} B e v \text {. }
$$

the cosmic ray intensity variation is not produced over all the latitude by the variation of cut-off energy.

In conclusion, the variation of cut-off energy can be solved analytically in case the static electric and magnetic field is symmetrical to the earth's magnetic dipole 
axis. The variation of cut-off energy caused by the static electric field can be determined approximately by the potential difference at the point where $r=1$ and $\lambda=0$, relative to the earth's potential, as long as the disturbance by the electric field is small.

\section{Appendix II Determination of Over-all Multiplicity}

(1) In case of the ionizing component

From Eq. (7), we can obtain the following relation

$$
m(E, x)=-\frac{1}{i(E)} \cdot \frac{d N(\lambda, x)}{d \lambda} \cdot \frac{d \lambda}{d E} .
$$

H.V. Neher ${ }^{(8)}$ obtained $m(E, x)$ at low energy region, using the primary spectrum in Eq. (6) and the vertical intensity of the ionizing component at various altitudes. The results are reproduced in Fig. 1 as the full lines. In the high energy region, although $m(E, x)$ cannot be obtained directly from observation, it is possible to obtain it approximately under the following considerations: As shown in Fig. 1 , in case $x$ is small, the full line has a critical energy $E_{c}(x)$, in the energy region larger than which $m(E, x)$ changes more slowly compared with that in the low energy region. In such a high energy region where $E \geq E_{c}(x)$, we assume that $m(E, x)$ is expressed by the following formula,

$$
m(E, x)=m_{c}(x)\left[\frac{E}{E_{c}(x)}\right]^{\alpha(x)},
$$

where $m_{c}(x)$ is the value of $m(E, x)$ in case $E=E_{c}(x)$. Introducing Eq. (A, 28) into Eq. (7), we obtain the following relation,

$$
N\left(\lambda_{c}, x\right)=\int_{E_{c}(x)}^{\infty} m_{c}(x)\left[\frac{E}{E_{c}(x)}\right]^{a(x)} \cdot i(E) d E, \quad \cdots \cdots \cdots
$$

where $\lambda_{c}$ is the latitude at which $E_{e}(x)$ becomes the cut-off energy. As $N\left(\lambda_{c}, x\right)$, $m_{c}(x), E_{c}(x)$ and $i(E)$ are known, $a(x)$ can be determined so as to satisfy Eq. (A, 29). The results obtained by such a procedure are shown in Fig. 1 and Table I, in case $x=150$ and 300 .

In case $x=1030$, although $E_{c}$ cannot be known directly from the observation, it seems to be reasonable to assume that the over-all multiplicity is similar to that in the case where $x$ is small, and that there also exists the critical energy $E_{c}$. If we adopt as $E_{c}$ the largest value of $E$ for which $m(E, 1030)$ can be obtained from the observation and assume that $m(E, 1030)$ is also given by Eq. $(A, 28)$ in the energy region where $E \geq E_{c}, a^{I}(1030)$ can be determined so as to satisfy Eq. (A,29) and gives the upper limit of $a(1030)$. The over-all multiplicity $m^{I}(E, 1030)$ obtained by such a procedure is shown in Fig. 1 and Table $I$. On the other hand, there is another way to determine $m(E, 1030)$ as follows; $a(x)$ in Eq. (A, 28) increases with the increase of $x$, so that if $a(300)$ is adopted as $a(1030)$, this gives the lower limit of $a(1030)$. In such a case, $E_{c}(1030)$ and $m_{c}(1030)$ can be determined so as to satisfy the following equation, 


$$
N(\lambda, 1030)=\int_{E_{\lambda}}^{E_{c}(1030)} m(E, 1030) i(E) d E+\int_{E_{c}(1030)}^{\infty} m_{c}(1030)\left[\frac{E}{E_{c}(1030)}\right]^{a(300)} \cdot i(E) d E, \quad(\mathrm{~A}, 30)
$$

where the over-all multiplicity obtained from the observation and its prolongation are used as $m(E, 1030)$ in the 1st term on the right hand side. The over-all multiplicity thus obtained is shown in Fig. 1 and Table $I$ with the notation $m^{I I}(E, 1030)$. The variations $y^{I}(\lambda, 1030, \Delta E, 0)$ and $y^{I I}(\lambda, 1030, \Delta E, 0)$ obtained by using $m^{I}(E, 1030)$ and $m^{I I}(E, 1030)$ give the lower and upper limits of $y$, respectively.

(2) In case of the neutron component

The latitude dependence curves of the neutron intensity used to obtain the over-all multiplicity are those of the Simpson observation ${ }^{(37),(38)}$ made at the atmospheric depthes $x=305$ and $x=680$. As these intensities are omnidirectional, the vertical intensities at $x=305$ can be obtained after the Gross transformation ${ }^{(38)}$ by using the absorption mean free path obtained by J.A. Simpson. ${ }^{(37)}$ But, in case $x=680$, it is not necessary to make the Gross transformation ${ }^{(18)}$ and here the latitude dependence curve normalized to unity at equator is adopted. Assuming that the over-all multiplicity is also expressed by Eq. $(\mathrm{A}, 28)$ in the high energy region, we can determine $a(x)$ in the same way, and the results are shown in Fig. 2 and Table I. The over-all multiplicities obtained here are different in form with those obtained by S.B. Treiman. ${ }^{(39)}$ This difference will be caused by the following facts that (1) the primary spectrums used are different from each other and (2) it is unreliable to determine $m$ at the energy region in the neighbourhood of $10 \mathrm{Bev}$. by observations only. But the variation $y$ at high latitude is almost not influenced by the adoptation of different over-all multiplicities, because the contribution of the high energy primary particles to the neutron intensity at high latitude is negligibly small as the latitude effect of the neutron component is very large.

(Read Oct. 30, 1953)

\section{References}

(1) K. Nagashima, Jour. Geomag. Geoelect., 3, 100 (1951).

(2) S. Chapman, Nature, 140, 425 (1937).

(3) H.V. Neher, V.Z. Peterson and E.A. Stern, Phys. Rev., 90, 655 (1953).

(4) S.E. Forbush, Rev. Mod. Phys., 11, 168 (1939).

(5) Y. Sekido, International Conference on Theoretical Physics, Symposium on Cosmic Rays, Tokyo, Japan (1953).

(6) W.H. Fonger, Phys. Rev., 91, 351 (1953).

(7) M.S. Vallarta and O. Godart, Rev. Mod. Phys., 11, 180 (1939).

(8) H.V. Neher, "Progress in Cosmic Ray Physics," North-Holland Publishing Co. Amsterdam, pp. 241-314 (1950).

(9) K. Dwight, Phys. Rev., 78, 40 (1950).

(10) Thanks are due to Mr. W.H. Fonger for pointing out author's inadquacy. In this paper, the expression of the Liouville's effect is corrected and used in a more accurate form than that which was adopted by W.H. Fonger. But the 
original expression in the previous paper ${ }^{(1)}$ holds good in the high energy region because $p c \approx E$.

(11) If we neglect the higher order of $\Delta E$ in Eq. (5), $y$ becomes to be proportional to $\Delta E$. But such an approximate formula of $y$ leads to the error which is nearly equal to that pointed out in Ref. (10), even if $\Delta E$ is small. Moreover, if $\Delta E$ becomes larger, this approximate formula is not correct and leads to a large error.

(12) The variation of the primary spectrum which is caused by the electric field not derived from potential will be discussed in $\S 5$.

(13) H. Alfvén, Phys. Rev., 72, 88 (1947).

(14) E.O. Kane, T.J.B. Shanely and J.A. Wheeler, Rev. Mod. Phys., 21, 51 (1949).

(15) B. Peters, "Progress in Cosmic Ray Physics," North-Holland Publishing Co. Amsterdam, p. 191 (1950).

(16) H.V. Neher, Phys. Rev., 83, 649 (1951).

(17) J.A. Van Allen and S.F. Singer, Phys. Rev., 78, 819 (1950).

J.R. Winckler, T. Stix, K. Dwight and R. Sabin, Phys. Rev., 79, 656 (1950).

(18) J.A. Simpson and W.C. Fagot, Phys. Rev., 90, 1068 (1953).

(19) D.H. Loughridge and P.F. Gast, Phys. Rev., 57, 937 (1940).

J.A. Simpson, Phys. Rev., 81, 639 (1951).

(20) L. Jànossy, Zeit. f. Phys., 104, 430 (1937).

(21) S. Yoshida and Y. Kamiya, Jour. Geomag. Geoelect., 5, 136 (1953).

(22) M. Wada, Report of Cosmic-Ray Laboratory, Scientific Research Institute (Japan) 1, 81 (1952).

(23) J.A. Simpson, W. Fonger and L. Wilcox, Phys. Rev., 85, 366 (1952).

(24) H.V. Neher and S.E. Forbush, Phys. Rev., 87, 889 (1952).

(25) S.E. Forbush, Terr. Mag., 43, 203 (1938).

(26) Attempts have been made by various authors to explain the diurnal variation of cosmic ray intensity by the electric field. See for example;

H. Elliot and D.W.N. Dolbear, J. Atmos. Terr. Phys., 1, 205 (1951).

Y. Sekido, S. Yoshida and Y. Kamiya, Rep. of Ionos. Res. in Japan, Vol. VI, No. 4, 195 (1952).

(27) Strictly speaking, if the electric field which is derived from potential is not symmetrical to the earth's magnetic dipole axis, the cut-off energy $E_{\lambda}$ varies with local time and then we may expect the diurnal variation of cosmic rays by such a field. As such a diurnal variation has the character similar to that expected from the static magnetic field hypothesis, ${ }^{(7)}$ we will consider later the former variation with the latter.

(28) L. Jànossy, “Cosmic Rays," Oxford, Clarendon Press, p. 268 (1950).

(29) W.F.G. Swann, Phys. Rev., 44, 224 (1933).

(30) The expression of $D$ in Ref. (28) is not correct. $D$ must be constant in the electro-magnetic field. ${ }^{(29)}$

(31) This problem wll be treated quantitatively in the near future. 
(32) D.I. Dawton and H. Elliot, J. Atmos. Terr. Phys., 3, 217 (1953).

J.A. Bergstralh and C.A. Schroeder, Phys. Rev., 81, 244 (1951).

(33) See for example, M.S. Vallarta, "On the Allowed Cone of Cosmic Radiation," Toronto Univ. Press (1938).

(34) Eqs. $(A, 8)$ and $(A, 9)$ are the expansion of the Störmer transformation in case of the existence of the static electric field.

(35) T. Nagata, J. Geophys. Res., 55, 127 (1950).

S. Hayakawa, J. Nishimura, T. Nagata and M. Sugiura, J. of Scien. Res. Inst. in Japan, 44, 121 (1950).

S.B. Treiman, Phys. Rev., 89, 130 (1953).

(36) J.A. Alpher, J. Geophys. Res., 55, 437 (1950).

(37) J.A. Simpson, Phys. Rev., 83, 1175 (1951).

(38) J.A. Simpson, W. Fonger and S.B. Treiman, Phys. Rev., 90, 934 (1953).

(39) S.B. Treiman, Phys. Rev., 86, 917 (1952).

The Meeting of the Society of Terrestrial Magnetism and Electricity : The 14th General Meeeting was held at the Kyoto University on Oct. 30-Nov. 1, 1953. 43 Reports were read, about 60 Members assembled.

The Tanakadate-Prize was awarded at the meeting for the following excellent worker :

The 14th, Mr. K. Nagashima, World-Wide Variation of Cosmic Ray Intensity by the Electro-Magnetic Fiẹd, 